QUARTERLY OF APPLIED MATHEMATICS

VOLUME LXIX, NUMBER 4

DECEMBER 2011, PAGES 691-721

S $0033-569 X(2011) 01256-3$

Article electronically published on June 29, 2011

\title{
FIELD BEHAVIOR NEAR THE EDGE OF A MICROSTRIP ANTENNA BY THE METHOD OF MATCHED ASYMPTOTIC EXPANSIONS
}

\author{
BY
}

A. BENDALI (University of Toulouse, INSA de Toulouse, Institut Mathématique de Toulouse, UMR CNRS 5219, Département de Génie Mathématique, 135 avenue de Rangueil F31077, Toulouse cedex 1 (France) and CERFACS, 42 Avenue Gaspard Coriolis, 31057 Toulouse Cedex 01 (France)),

A. MAKHLOUF (University of Toulouse, INSA de Toulouse, Institut Mathématique de Toulouse, UMR CNRS 5219, Département de Génie Mathématique, 135 avenue de Rangueil F31077, Toulouse cedex 1 (France)),

AND

S. TORDEUX (Project-team Magique-3D, INRIA Bordeaux Sud-Ouest. LMA Pau, CNRS UMR

5142, I.P.R.A., Université de Pau et des Pays de l'Adour, avenue de l'Université BP 1155, 64013 PAU Cedex (France) and CERFACS, 42 Avenue Gaspard Coriolis, 31057 Toulouse Cedex 01 (France))

Abstract. The cavity model is a wide-spread powerful empirical approach for the numerical simulation of microstrip antennas. It is based on several hypotheses assumed a priori: a dimension reduction in the cavity, that is, the zone limited by a metallic patch and the ground plane in which is fed the antenna, supplied by the additional condition that the open sides of the cavity act as magnetic walls. An additional important assumption of this model consists in an adequate description of the singular field behavior in the proximity of the edge of the patch. A simplified two-dimensional problem incorporating the main features of the field behavior near the edge of the patch and inside the cavity is addressed. The method of matched asymptotic expansions is used to carry out a two-scale asymptotic analysis of the field relative to the thickness of the cavity. All the empirical hypotheses at the basis of the derivation of the cavity model can thus be recovered. Proved error estimates are given in a simplified framework where the dielectric constants of the substrate are assumed to be 1 in order to avoid some unimportant technical difficulties.

Received March 18, 2010.

2000 Mathematics Subject Classification. Primary 34E05, 35Q60, 81T80.

E-mail address: abendali@insa-toulouse.fr

E-mail address: abdelkader.makhlouf@gmail.com

E-mail address: stordeux@insa-toulouse.fr 


\section{Introduction.}

1.1. Basic modeling methods for patch antennas. A rectangular microstrip antenna, also called a patch antenna, consists of a single rectangular metal patch placed at a small distance above a ground plane (see Figure 1).

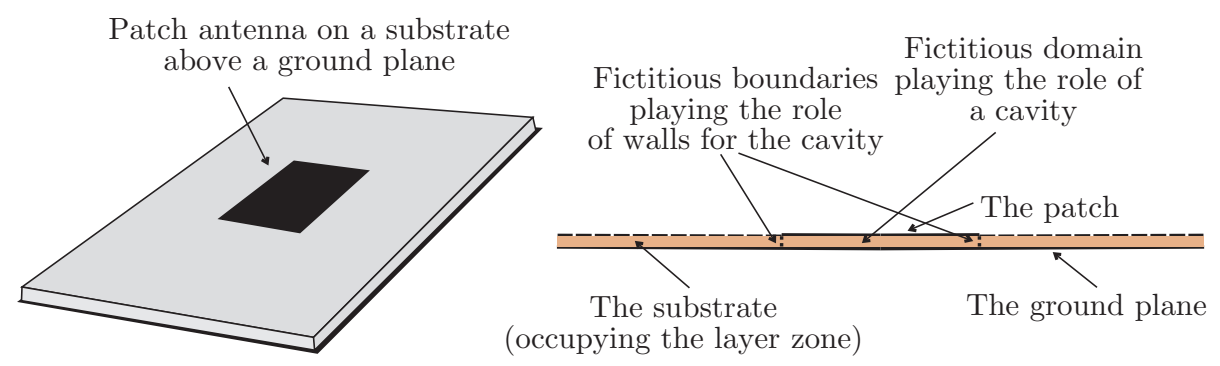

FIG. 1. A rectangular microstrip antenna and its cross-sectional view

The patch is posed above a dielectric slab of constant thickness, called the substrate in this context, covering a ground plane; see, for instance, [1, 3. In the terminology of microstrip antennas, the zone located between the patch and the ground plane is called the cavity (see Figure 1). Generally a probe is placed inside the cavity and is used for recovering the field received by the antenna or for feeding it in the transmitting mode.

The directional efficiency of an antenna, both as a receiving or a transmitting device, is characterized by its radiation pattern (see, e.g., [1). Only the transmitting mode is thus relevant when the objective is to determine this pattern. Several modeling methods can be used to do this (see, e.g., 1, 3]). The full-wave models are the most straightforward and consist in a direct numerical solution of the Maxwell equations complemented by appropriate transmission and boundary conditions (see, e.g., [15, 16, 8, 11, 31, 28]). However, in the opinion of the authors, the accuracy of the full-wave methods when applied for this kind of problem is highly questionable since it seems hard for a direct numerical simulation based on either a finite difference, a finite element, or a boundary element method to correctly reproduce either the singular behavior of the electromagnetic field near an edge of the patch or the dimensional reduction occurring inside the cavity. From an empirical standpoint, the transmission-line model represents the patch as two parallel radiating slots on a ground plane corresponding to the open sides of the cavity along the width of the patch. Another empirical approach, the cavity model, can be somehow considered as an elaborate extension of the transmission-line model and generally results in a more accurate determination of the electromagnetic field. For this model also, the radiated field is produced by the radiating slots involved in the transmission-line model. By taking into account the singular behavior of such a kind of electromagnetic field a priori, these two methods result in an ease of solving and an accuracy which, in the opinion of the authors, can be reached by a plain numerical approach only with effort and difficulty (cf., e.g., 1, 3, 22]). Our purpose in this study is to obtain some preliminary mathematical justifications of these empirical models derived from the exact general equations governing the underlying radiation problem. The kernel of this derivation lies in a sharp determination of the behavior of the field in the neighborhood 
of an edge related to a radiating side of the cavity. This point constitutes the main topic addressed in this paper.

The authors would like to acknowledge the anonymous referee who drew our attention to some previous references [25, 24, 23, where a first-order correction of the magnetic wall condition has been given in terms of an approximate impedance boundary condition. In some way, the derivation of such a kind of boundary condition can be seen as a justification of the magnetic wall assumption of the cavity model. These boundary conditions and, as a result, the underlying justification of the magnetic wall hypothesis thus provided, are established only under the a priori assumption that in the proximity of the edge the electromagnetic field is static. Actually, the first justification of the cavity model in a simplified but representative situation such as the one considered in this work was obtained in [19] in the form of a first-order thin-dielectric substrate approximation of the reflection coefficient of a TEM mode under the patch obliquely incident on the edge. The approximation is derived from clever estimates of the analytical expressions obtained in 4] which do not yield the electromagnetic field explicitly but only in terms of some Sommerfeld integrals obtained through a Wiener-Hopf procedure. This is why in our opinion only a partial justification of the cavity model is carried out in the former reference with in particular no mention of the fact that the radiated field is close in this context to that of a monopole placed at the edge above a ground plane. Below we show how this justification can be completed in a formal way, thus yielding a firstorder expansion of the field outside a boundary layer around the edge of the patch. This will enable us to assess the first-order part of the asymptotic expansion obtained here through a completely different procedure. However, none of these references provides a second-order expansion and gives a rigorous and complete justification of the cavity model based on error estimates such as is done in this work.

1.2. A simplified problem. The full three-dimensional problem still seems out of reach, at least for the level of justification intended to be given here. We are interested in this work mainly in the behavior of the field in the proximity of a radiating slot, meanwhile neglecting the influence of the other edge. In a quite natural way, we are thus led to consider the geometry related to the following domain with a straight boundary for representing the infinite ground plane, an edge of the patch, and the cavity (see Figure 2):

$$
\left.\left.\Omega^{\delta}=\left\{(x, y) \in \mathbb{R}^{2}: y>-\pi \delta\right\} \backslash\{]-\infty, 0\right] \times\{0\}\right\} .
$$

The decomposition of $\Omega^{\delta}$ into the following three nonoverlapping subdomains

$$
\begin{aligned}
\Omega & =\left\{(x, y) \in \mathbb{R}^{2}: y>0\right\}, \\
\Omega_{\mathrm{C}}^{\delta} & =\left\{(x, y) \in \mathbb{R}^{2}: x<0 \text { and }-\pi \delta<y<0\right\}, \\
\Omega_{\mathrm{L}}^{\delta} & =\left\{(x, y) \in \mathbb{R}^{2}: x>0 \text { and }-\pi \delta<y<0\right\}
\end{aligned}
$$

is appropriate for describing some physical features of the problem, in particular those on which is based the cavity model. It will also be important for stating some mathematical properties owned by its solution. The "half-plane" $\Omega$ is the domain in which the wave is propagating. The cavity $\Omega_{\mathrm{C}}^{\delta}$ is assumed to be semi-infinite since we focus only on the behavior of the field near one edge of the patch. It will also be helpful to consider the 


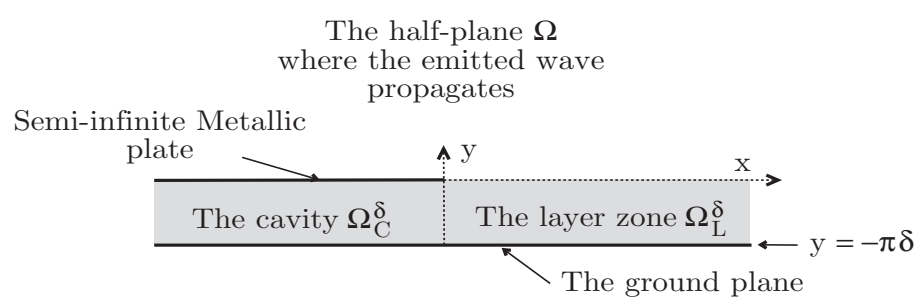

FIG. 2. Two-dimensional geometry of the radiation problem

domain corresponding to the dielectric slab

$$
\Omega_{\mathrm{S}}^{\delta}=\left\{(x, y) \in \mathbb{R}^{2}:-\pi \delta<y<0\right\} .
$$

The small distance separating the patch from the ground plane is normalized to $\pi \delta$ to simplify some calculations carried out below.

As is well known, no H-polarized wave (a two-dimensional wave whose electric field is directed along the direction where the electromagnetic field is constant) can propagate inside $\Omega_{\mathrm{C}}^{\delta}$. It is thus natural to assume that the field is an E-polarized wave, which is described by a single scalar function $u^{\delta}$ giving the only nonzero component of the magnetic field, directed once along the direction where the geometry is not varying.

In order to avoid difficulties which are unimportant in this study, we furthermore suppose that the wave number $\kappa$ characterizing the ambient medium corresponds to an absorbing material, that is,

$$
\kappa=|\kappa| \exp (i \arg \kappa), \quad 0<\arg \kappa<\pi / 2 .
$$

The contrast properties of the dielectric are then described by two complex numbers respectively characterizing its electric permittivity and magnetic permeability relative to the ambient medium

$$
\varepsilon=\varepsilon_{r}+i \varepsilon_{i}, \mu=\mu_{r}+i \mu_{i} \text { with } \varepsilon_{r}, \mu_{r} \geq 1 \text { and } \varepsilon_{i}, \mu_{i} \geq 0 .
$$

Conveniently we shall also make use of the refractive index and the relative impedance of the substrate, respectively

$$
n_{\mathrm{s}}=\sqrt{\varepsilon \mu}, \zeta_{\mathrm{s}}=\sqrt{\mu / \varepsilon},
$$

to refer to its dielectric constants.

Letting $\kappa_{\mathrm{s}}=\kappa \sqrt{\varepsilon \mu}$ be the wave number in the substrate, we directly get that only two modes, $\exp \left(i \kappa_{\mathrm{s}} x\right)$ and $\exp \left(-i \kappa_{\mathrm{s}} x\right)$, can "propagate" within the cavity since $\delta$ is destined to tend to zero. The latter describes the wave being reflected by the opening of the cavity, while the former can be used as a way of feeding the antenna as it is done here. To be able to state the boundary-value problem satisfied by $u^{\delta}$, we introduce the following functional setting. Since $u^{\delta}$ behaves as $\exp \left(i \kappa_{\mathrm{s}} x\right)$ at the back of the cavity, that is, for $x$ tending to $-\infty$, it is sought in the following Fréchet space generally used to describe the regularity properties of a time-harmonic wave on the bounded subsets of the solution domain (cf., e.g., [37])

$$
\mathrm{H}_{\mathrm{loc}}^{1}\left(\overline{\Omega^{\delta}}\right)=\left\{v \in \mathcal{D}^{\prime}\left(\Omega^{\delta}\right): \varphi v \in \mathrm{H}^{1}\left(\Omega^{\delta}\right), \forall \varphi \in \mathcal{D}\left(\Omega^{\delta}\right)\right\} .
$$


To describe how the propagative mode $x \rightarrow \exp \left(i \kappa_{\mathrm{s}} x\right)$ is used to feed the "antenna", we consider the following cutoff function built as follows. Let $c_{-}<c_{+}<0$ and $x \rightarrow c(x)$ be respectively two real fixed negative numbers and a smooth nonincreasing real function such that $c(x)=1$ for $x<c_{-}$and $c(x)=0$ for $x>c_{+}$. Denoting by $1_{]-\pi \delta, 0[}$ the characteristic function of the interval $]-\pi \delta, 0\left[\right.$, we directly get that $(x, y) \rightarrow f^{\delta}(x, y)=$ $c(x) \exp \left(i \kappa_{\mathrm{s}} x\right) 1_{]-\pi \delta, 0[}(y)$ is a function in $\mathcal{C}^{\infty}\left(\overline{\Omega^{\delta}}\right)$ whose support is contained in the cavity $\Omega_{\mathrm{C}}^{\delta}$ which differs there from $\exp \left(i \kappa_{\mathrm{s}} x\right)$ on a bounded subset only. We can then state the boundary-value problem satisfied by $u^{\delta}$ :

$$
\begin{cases}u^{\delta} \in \mathrm{H}_{\mathrm{loc}}^{1}\left(\overline{\Omega^{\delta}}\right), & \\ \Delta u^{\delta}+\kappa^{2} u^{\delta}=0, & \text { in } \Omega, \\ \Delta u^{\delta}+\kappa_{\mathrm{s}}^{2} u^{\delta}=0, & \text { in } \Omega_{\mathrm{S}}^{\delta}, \\ \partial_{y} u^{\delta}\left(x, 0^{ \pm}\right)=0, & \forall x<0, \\ \partial_{y} u^{\delta}(x,-\pi \delta)=0, & \forall x \in \mathbb{R}, \\ \varepsilon^{-1} \partial_{y} u^{\delta}\left(x, 0^{-}\right)=\partial_{y} u^{\delta}\left(x, 0^{+}\right), & \forall x>0, \\ u^{\delta}-f^{\delta} \in \mathrm{H}^{1}\left(\Omega^{\delta}\right) . & \end{cases}
$$

The following variational formulation yields an adequate mathematical framework for problem (1.10):

$$
u^{\delta} \in \mathrm{H}_{\mathrm{loc}}^{1}\left(\overline{\Omega^{\delta}}\right): u^{\delta}-f^{\delta} \in \mathrm{H}^{1}\left(\Omega^{\delta}\right), \quad a^{\delta}\left(u^{\delta}-f^{\delta}, v\right)=\ell^{\delta} v, \quad \forall v \in \mathrm{H}^{1}\left(\Omega^{\delta}\right) .
$$

In particular, the existence and the uniqueness of $u^{\delta}$ follow from a straightforward application of the Lax-Milgram lemma. Conveniently we do not distinguish between the constants $1 / \varepsilon$ and $\mu$ and the functions defined almost everywhere on $\Omega^{\delta}$ by 1 on $\Omega$ and $\varepsilon^{-1}$ and $\mu$ respectively on $\Omega_{\mathrm{S}}^{\delta}$. The involved bilinear and linear forms on $\mathrm{H}^{1}\left(\Omega^{\delta}\right)$ can then be defined by

$$
\begin{aligned}
a^{\delta}(u, v) & =\int_{\Omega^{\delta}}\left(\frac{1}{\varepsilon} \nabla u(x, y) \cdot \nabla v(x, y)-\kappa^{2} \mu u(x, y) v(x, y)\right) d x d y \\
\ell^{\delta} v & =\int_{\Omega_{\mathrm{C}}^{\delta}} \varepsilon^{-1}\left(c^{\prime \prime}(x)+2 i \kappa_{\mathrm{s}} c^{\prime}(x)\right) \exp \left(i \kappa_{\mathrm{s}} x\right) v(x, y) d x d y
\end{aligned}
$$

respectively. Moreover, the consideration of an absorbing medium, characterized by condition (1.6), readily yields the following stability estimate:

$$
\left\|u^{\delta}-f^{\delta}\right\|_{H^{1}\left(\Omega^{\delta}\right)} \leq C \sup _{\|v\|_{H^{1}\left(\Omega^{\delta}\right)} \leq 1}\left|\ell^{\delta} v\right|,
$$

where $C$ is a constant independent of $\delta$ and of the particular linear form $\ell^{\delta}$.

Throughout this paper, any function of the complex variable requiring a determination for the argument, such as the previous square root or the logarithm with a complex argument, is implicitly assumed to correspond to its principal branch, that associated with a cut along the negative real axis. The usual notation in the theory of partial differential equations, including Sobolev spaces, is used without further comment (cf., e.g., [33, 5]). 
In addition to enabling a relevant description of the behavior of the electromagnetic field near a radiating slot of a rectangular patch antenna, problem (1.10) is similar to the one corresponding to the scattering of an acoustic wave by a parallel-walled duct considered by McIver and Rawlins [27. The two-scale asymptotic expansion developed by these authors, as well as some of the tools they considered to construct it, will be at the basis of the justification carried out here. We however depart from their study in pushing the asymptotic expansion to the next order, namely to the second-order one, and in considering that the dielectric slab $\Omega_{\mathrm{S}}^{\delta}$ has a contrast relative to the ambient medium. We also show how the second-order expansion makes it possible to establish error estimates for the approximation corresponding to the cavity model. These estimates thus complete the mathematical justification of this model. Although the error estimates are established for the simplified case where the dielectric constants of the slab are assumed to be 1 to avoid unnecessary technicalities based on the Mellin transform, the procedure for constructing the asymptotic expansions clearly indicates the adaptations necessary for general substrates. Actually, it is enough to replace a semi-explicit function defined in terms of the inverse of a conformal mapping by a completely implicit one defined as a determined solution to a boundary-value problem given below.

1.3. Outline of the paper. The paper is organized as follows. In Section 2 we start with a rapid presentation of an adaptation of the results of [19] to problem (1.10) and complete it with respect to the part of $u^{\delta}$ radiated in the upper-plane $\Omega$. The construction of the second-order matched asymptotic expansions for $u^{\delta}$ is next obtained through a formal mathematical approach. However, the existence of these expansions is established rigorously by proving that all the boundary-value problems satisfied by their various terms are well-posed and that the matching requirements at different scales are all fulfilled. Section 3 is then devoted to obtaining error estimates, in a simplified framework as said above, which then rigorously validate the formal approach.

\section{Existence of the two-scale asymptotic expansion.}

2.1. First-order thin-substrate approximation from a Wiener-Hopf solution. An elementary separation of variables within the cavity $\Omega_{\mathrm{C}}^{\delta}$ clearly shows that

$$
u^{\delta}(x, y)=e^{i \kappa_{\mathrm{s}} x}+R^{\delta} e^{-i \kappa_{\mathrm{s}} x}+\sum_{m=1}^{\infty} R_{m}^{\delta} e^{x \sqrt{m^{2} / \delta^{2}-\kappa_{\mathrm{s}}^{2}}} \cos (m y / \delta)
$$

for $x<0$ and $-\pi<y<0$ making it possible to identify the reflection coefficient as $R^{\delta}$. To adapt the results in [19 to the present framework, we substitute the notation within this reference and [4] for the setting of problem (1.10) as follows:

$$
i \rightarrow-i, \alpha \rightarrow 0, \Gamma \rightarrow-R^{\delta}, d \rightarrow \pi \delta, y \rightarrow-x, z \rightarrow y+\delta \pi, k_{0} \rightarrow \kappa .
$$

According to [4], $u^{\delta}$ has the following integral representation outside the substrate:

$$
u^{\delta}(x, y)=-i \kappa \zeta_{\mathrm{s}} \delta \int_{-\infty}^{+\infty} e^{i\left(f_{e}(-\lambda)+f_{e}\left(-i n_{\mathrm{s}}\right)\right) / 2} \frac{e^{-\kappa u_{0}(\lambda) y-i \kappa \lambda x}}{u_{0}(\lambda)} d \lambda \text { for } y>0,
$$


where $u_{0}(\lambda)=\sqrt{\lambda^{2}-1}$ and in which we shall make use only of the following approximation of $f_{e}$ :

$$
f_{e}(w)=\frac{\kappa \delta}{\varepsilon}\left(\begin{array}{c}
\frac{u_{s}^{2}(w)}{u_{0}(w)}\left(\begin{array}{c}
\ln \left(w+u_{0}(w)\right)- \\
\ln \left(w-u_{0}(w)\right)
\end{array}\right)+ \\
2 w(\ln (-i \kappa \pi \delta)+\gamma-1)+ \\
2 \varepsilon w\left(2 Q_{0}\left(\frac{1-\varepsilon}{1+\varepsilon}\right)-\ln 2 \pi\right)
\end{array}\right)+O\left((\kappa \delta w)^{2} \ln \kappa \delta\right)
$$

established in [19]. In this formula,

$$
u_{\mathrm{s}}(w)=\sqrt{w^{2}-n_{\mathrm{s}}^{2}}, Q_{0}(z)=\sum_{m=1}^{\infty} z^{m} \ln m(|z|<1)
$$

and $\gamma$ is the Euler constant $0.5772 \ldots$ A first-order approximation of $u^{\delta}$ for $y>0$ can hence be obtained in a formal way by

$$
\begin{aligned}
u^{\delta}(x, y) & =-i \kappa \zeta_{\mathrm{s}} \delta \int_{-\infty}^{+\infty} \frac{e^{-\kappa u_{0}(\lambda) y-i \kappa \lambda x}}{u_{0}(\lambda)} d \lambda+o(\delta) \\
& =-i \kappa \zeta_{\mathrm{s}} \delta \int_{-\infty}^{+\infty} \frac{e^{-\sqrt{\sigma^{2}-\kappa^{2}} y-i \sigma x}}{\sqrt{\sigma^{2}-\kappa^{2}}} d \sigma+o(\delta) .
\end{aligned}
$$

The last integral can be calculated explicitly (cf. [32, p. 32]) yielding

$$
u^{\delta}(x, y)=\kappa \pi \delta \zeta_{\mathrm{s}} H_{0}^{(1)}(\kappa r)+o(\delta) .
$$

The usual polar coordinates are denoted by $(r, \theta)$, and $H_{\ell}^{(1)}$ is the Hankel function of the first kind of positive integer order $\ell$ (cf., e.g., [36, 20, 35]). We thus get the first-order approximation of $u^{\delta}$ in $\Omega$ by a monopole placed above a ground plane announced in the introduction. It is however worth mentioning that the above formal procedure, by missing the remainder term $O\left((\kappa \delta \lambda)^{2} \ln \kappa \delta\right)$ for large values of $\lambda$ in the integral, rubs out some boundary layer terms correcting (2.7), which are concentrated in the proximity of the edge as the two-scale asymptotic expansion given below will show.

The approximation of the reflection coefficient does not face the difficulty raised by the large values of the argument of $f_{e}(w)$. It was given in [19] in the following form:

$$
R^{\delta}=-e^{i f_{e}\left(-n_{\mathrm{s}}\right)} \approx-\exp \left(-2 i \kappa_{\mathrm{s}} \delta\left(\begin{array}{c}
\frac{1}{\varepsilon}(\ln (-i \kappa \delta \pi)+\gamma-1) \\
+2 Q_{0}\left(\frac{1-\varepsilon}{1+\varepsilon}\right)-\ln 2 \pi
\end{array}\right)\right)
$$

but can also be expressed by means of the following first-order expansion:

$$
R^{\delta}=-1+\kappa \pi \zeta_{\mathrm{s}} \delta\left(\begin{array}{c}
1+\frac{2 i}{\pi}\left(\ln \frac{\kappa \delta}{2}+\gamma\right) \\
-\frac{2 i}{\pi}\left(1-2 \varepsilon Q_{0}\left(\frac{1-\varepsilon}{1+\varepsilon}\right)+(\varepsilon-1) \ln 2 \pi\right)
\end{array}\right)+o(\delta) .
$$

2.2. The form of the asymptotic expansion. The small width $\pi \delta$ of $\Omega_{\mathrm{C}}^{\delta}$ is at the basis of the functioning of a patch antenna. This physical feature gives rise to a dimensional reduction; namely, here the wave is one-dimensional by nature inside $\Omega_{\mathrm{C}}^{\delta}$. On the other hand, this one-dimensional solution has to be matched with a two-dimensional wave outside the cavity. As is well known for such problems (see, for instance, 27, 18]), the two kinds of solutions have to be linked each to the other in a zone, having a small size, around the opening of $\Omega_{\mathrm{C}}^{\delta}$, called a boundary layer. To mathematically deal in 
a convenient way with this zone, we assume that it is infinitesimally small as usual in this kind of multi-scale problems (see, e.g., [14, 26, 27, 18]). Following the two-scale asymptotic expansion devised by McIver and Rawlins for a similar problem [27, we look for an expansion of $u^{\delta}$ in the following form outside the boundary layer:

$$
u^{\delta}(x, y)= \begin{cases}\sum_{n=0}^{N} \sum_{m=0}^{n} \delta^{n} \ln ^{m} \delta u^{n, m}(x, y)+\delta^{N} \varepsilon_{\delta} & \text { for }(x, y) \in \Omega, \\ \left.\sum_{n=0}^{N} \sum_{m=0}^{n} \delta^{n} \ln ^{m} \delta u_{\mathrm{C}}^{n, m}(x, Y)\right|_{Y=y / \delta}+\delta^{N} \varepsilon_{\delta} & \text { for } x<0 \text { and } \\ \left.\sum_{n=0}^{N} \sum_{m=0}^{n} \delta^{n} \ln ^{m} \delta u_{\mathrm{L}}^{n, m}(x, Y)\right|_{Y=y / \delta}+\delta^{N} \varepsilon_{\delta} & \text { for } x>0 \text { and } \\ & -\pi<Y<0,\end{cases}
$$

and "inside" the boundary layer,

$$
u^{\delta}(\delta X, \delta Y)=\sum_{n=0}^{N} \sum_{m=0}^{n} \delta^{n} \ln ^{m} \delta \Pi^{n, m}(X, Y)+\delta^{N} \varepsilon_{\delta} \text { for }(X, Y) \in \widehat{\Omega},
$$

where

$$
\left.\left.\widehat{\Omega}=\left\{(X, Y) \in \mathbb{R}^{2}: Y>-\pi\right\} \backslash\{]-\infty, 0\right] \times\{0\}\right\}
$$

is the domain in which the fast variables $X=x / \delta$ and $Y=y / \delta$ are varying "as $\delta \rightarrow$ 0 ". 1 Hereafter, $\varepsilon_{\delta}$ denotes a generic term such that $\varepsilon_{\delta} \rightarrow 0$ when $\delta \rightarrow 0$. In the same way, any coefficient, corresponding to a pair of integers $(n, m)$ outside the range $0 \leq m \leq n$, is assumed to be zero. Expansions (2.10) and (2.11) are respectively called the "outer" and the "inner" expansions. As usual in the treatment of these kinds of two-scale problems, "inner" and "outer" will be used for referring to these expansions without further comment.

2.3. Characterization of the solutions to the outer problems.

2.3.1. The outer problems. Some function spaces are needed to set the equations satisfied by the coefficients of the outer expansions. The choice of these spaces is based on the following feature. The solution is globally in $\mathrm{H}^{1}\left(\Omega^{\delta}\right)$. The coefficients $u^{n, m}, u_{\mathrm{L}}^{n, m}$ and $u_{\mathrm{C}}^{n, m}$ of the outer expansions inherit this regularity, but only outside any neighborhood of $(0,0)$ since their behavior for $(x, y) \rightarrow(0,0)$ cannot be restrained from any information which would be available a priori. Consequently they are respectively sought in the Fréchet spaces defined as follows. We shall say that a distribution $v \in \mathcal{D}^{\prime}(\Omega)$ is in $\mathrm{H}_{*}^{1}(\Omega)$ if $\left(1-\varphi \otimes_{x, y} \varphi\right) v \in \mathrm{H}^{1}(\Omega)$ for all $\varphi \in \mathcal{D}(\mathbb{R})$ such that $\exists r>0, \varphi(x)=1$ for $|x|<r$. Similarly one defines $\mathrm{H}_{*}^{1}\left(\widehat{\Omega}_{\mathrm{C}}\right)$ and $\mathrm{H}_{*}^{1}\left(\widehat{\Omega}_{\mathrm{L}}\right)$ by requiring that $\left(1-\varphi \otimes_{x, Y} 1\right) v \in \mathrm{H}^{1}\left(\widehat{\Omega}_{\mathrm{C}}\right)$ and $\left(1-\varphi \otimes_{x, Y} 1\right) v \in \mathrm{H}^{1}\left(\widehat{\Omega}_{\mathrm{L}}\right)$ respectively. In these definitions, $\widehat{\Omega}_{\mathrm{C}}$ and $\widehat{\Omega}_{\mathrm{L}}$ are the respective scaled representations of the cavity and the layer

$$
\begin{aligned}
& \widehat{\Omega}_{\mathrm{C}}=\left\{(x, Y) \in \mathbb{R}^{2}: x<0 \text { and }-\pi<Y<0\right\}, \\
& \widehat{\Omega}_{\mathrm{L}}=\left\{(x, Y) \in \mathbb{R}^{2}: x>0 \text { and }-\pi<Y<0\right\} .
\end{aligned}
$$

\footnotetext{
${ }^{1}$ For this straight geometry, $\widehat{\Omega}$ is nothing else but a scaled representation of $\Omega^{\delta}$. For the case of a curved geometry, $\widehat{\Omega}$ will be a straight and scaled geometry tangent to the curved one.
} 
Plugging (2.10) into (1.10), we readily get that the coefficients involved in the outer expansions satisfy the following system of equations:

$$
\begin{aligned}
& \left\{\begin{array}{l}
u^{n, m} \in \mathrm{H}_{*}^{1}(\Omega), \\
\Delta u^{n, m}(x, y)+\kappa^{2} u^{n, m}(x, y)=0 \text { in } \Omega, \\
\partial_{y} u^{n, m}(x, 0)=0 \text { for } x<0,
\end{array}\right. \\
& \begin{cases}u_{\mathrm{L}}^{n, m} \in \mathrm{H}_{*}^{1}\left(\widehat{\Omega}_{\mathrm{L}}\right), & \\
\partial_{Y}^{2} u_{\mathrm{L}}^{n+2, m}(x, Y)=-\left(\partial_{x}^{2}+\kappa_{\mathrm{s}}^{2}\right) u_{\mathrm{L}}^{n, m}(x, Y) & \text { in } \widehat{\Omega}_{\mathrm{L}}, \\
\partial_{Y} u_{\mathrm{L}}^{n, m}(x,-\pi)=0 & \text { for } x>0,\end{cases} \\
& \left\{\begin{array}{cl}
u_{\mathrm{C}}^{0,0}-\exp \left(i \kappa_{\mathrm{S}} x\right) \in \mathrm{H}_{*}^{1}\left(\widehat{\Omega}_{\mathrm{C}}\right), & \\
u_{\mathrm{C}}^{n, m} \in \mathrm{H}_{*}^{1}\left(\widehat{\Omega}_{\mathrm{C}}\right) & \text { for }(n, m) \neq(0,0), \\
\partial_{Y}^{2} u_{\mathrm{C}}^{n+2, m}(x, Y)= & \text { in } \widehat{\Omega}_{\mathrm{C}}, \\
\quad-\left(\partial_{x}^{2}+\kappa_{\mathrm{s}}^{2}\right) u_{\mathrm{C}}^{n, m}(x, Y) & \text { for } x<0, \\
\partial_{Y} u_{\mathrm{C}}^{n, m}(x,-\pi)=0 & \text { for } x<0, \\
\partial_{Y} u_{\mathrm{C}}^{n, m}(x, 0)=0 &
\end{array}\right. \\
& \begin{cases}u^{n, m}(x, 0)=u_{\mathrm{L}}^{n, m}(x, 0) & \text { for } x>0, \\
\partial_{y} u^{n, m}(x, 0)=\frac{1}{\varepsilon} \partial_{Y} u_{\mathrm{L}}^{n+1, m}(x, 0) & \text { for } x>0 .\end{cases}
\end{aligned}
$$

2.3.2. Partial determination of the expansions inside the cavity and the layer. A partial determination of the coefficients of the outer asymptotic expansions inside the cavity and the layer is embodied in the following lemma.

LEMma 2.1. The coefficients $u_{\mathrm{L}}^{n, m}$ and $u^{n, m}$ satisfy

$$
\begin{aligned}
& u_{\mathrm{L}}^{n, m}(x, Y)=u^{n, m}(x, 0) \\
& \quad-\left(\partial_{x}^{2}+\kappa_{\mathrm{s}}^{2}\right)\left(Y \int_{-\pi}^{0} u_{\mathrm{L}}^{n-2, m}(x, s) d s+\int_{0}^{Y}(Y-s) u_{\mathrm{L}}^{n-2, m}(x, s) d s\right), \\
& \partial_{y} u^{n, m}(x, 0)=-\frac{1}{\varepsilon}\left(\partial_{x}^{2}+\kappa_{\mathrm{s}}^{2}\right) \int_{-\pi}^{0} u_{\mathrm{L}}^{n-1, m}(x, s) d s,
\end{aligned}
$$

respectively in $\widehat{\Omega}_{\mathrm{L}}$ and for $x>0$. In particular, if $u_{\mathrm{L}}^{n-1, m}$ is independent of $Y$, then

$$
\begin{aligned}
& u_{\mathrm{L}}^{n+1, m}(x, Y)=u^{n+1, m}(x, 0)-Y(Y / 2+\pi)\left(\partial_{x}^{2}+\kappa_{\mathrm{s}}^{2}\right) u^{n-1, m}(x, 0), \\
& \partial_{y} u^{n, m}(x, 0)=-\frac{\pi}{\varepsilon}\left(\partial_{x}^{2}+\kappa_{\mathrm{s}}^{2}\right) u^{n-1, m}(x, 0) \text { for } x>0 .
\end{aligned}
$$

The determination of $u_{\mathrm{C}}^{n, m}$ is reduced to that of a (reflection) coefficient $R^{n, m}$ :

$$
\begin{aligned}
& u_{\mathrm{C}}^{0,0}=\exp \left(i \kappa_{\mathrm{s}} x\right)+R^{0,0} e^{-i \kappa_{\mathrm{s}} x}, \\
& u_{\mathrm{C}}^{n, m}(x, Y)=R^{n, m} e^{-i \kappa_{\mathrm{s}} x} \text { if } n \neq 0 .
\end{aligned}
$$

Proof. The expressions of $u_{\mathrm{L}}^{n, m}$ and $\partial_{y} u^{n, m}(x, 0)$ in (2.19) result from a straightforward integration of (2.16) considered as a second-order differential equation with respect to $Y$ and using the transmission conditions (2.18). The simpler expressions (2.20) are then an 
obvious particularization of (2.19). To deal with the cavity equations, we first decompose $u_{\mathrm{C}}^{n, m}$ as follows:

$$
u_{\mathrm{C}}^{n, m}(x, Y)=\dot{u}_{\mathrm{C}}^{n, m}(x)+w_{\mathrm{C}}^{n, m}(x, Y), \quad \int_{-\pi}^{0} w_{\mathrm{C}}^{n, m}(x, Y) d y=0 \quad \forall x<0 .
$$

Integrating (2.17) with respect to $Y$, we get

$$
\left(\partial_{x}^{2}+\kappa_{\mathrm{s}}^{2}\right) \dot{u}_{\mathrm{C}}^{n, m}(x)=0, \quad \forall x<0 .
$$

It is then an easy matter to show by induction that $w_{\mathrm{C}}^{n, m}=0$, which hence yields (2.21).

REMARK 2.2. The form found for the coefficients $u_{\mathrm{C}}^{n, m}$ in the above lemma clearly brings out the dimensional reduction of the field inside the cavity.

2.3.3. Characterization of the solutions to the outer problems in the half-plane. The coefficient $u^{n, m}$ satisfies the following nonstandard boundary-value problem:

$$
\begin{cases}u^{n, m} \in \mathrm{H}_{*}^{1}(\Omega), & \\ \Delta u^{n, m}+\kappa^{2} u^{n, m}=0, & \text { in } \Omega, \\ \partial_{y} u^{n, m}(x, 0)=0, & \forall x<0, \\ \partial_{y} u^{n, m}(x, 0)=-\frac{1}{\varepsilon}\left(\partial_{x}^{2}+\kappa_{\mathrm{S}}^{2}\right) \int_{-\pi}^{0} u_{\mathrm{L}}^{n-1, m}(x, s) d s, & \forall x>0 .\end{cases}
$$

The Neumann data of this problem will be available at the time of determining $u^{n, m}$ from the second relation in (2.19). Moreover, as will be established below, $\partial_{y} u^{n, m}(x, 0)$ is $\mathcal{C}^{\infty}$ for $x>0$, adequately decreases when $x \rightarrow \infty$ in order to be compatible with the behavior of $u^{n, m}$ as a function in $\mathrm{H}_{*}^{1}(\Omega)$, and has a singularity when $x \rightarrow 0$ which can be described in terms of the functions $x^{\alpha} \ln ^{\beta} x, \alpha, \beta \in \mathbb{R}, \beta \geq 0$. Extending a previous result of Tordeux 34 for the case of a homogeneous Neumann boundary condition, Makhlouf established in 22. that $u^{n, m}$ is known up to a finite number of nonzero constants $C_{\ell}^{n, m}$ such that

$$
u^{n, m}=w^{n, m}+\sum_{\ell=0}^{+\infty} C_{\ell}^{n, m} H_{\ell},
$$

where $H_{\ell}$ is the multipole created by sources concentrated at $(0,0)$,

$$
H_{\ell}(r, \theta)=H_{\ell}^{(1)}(\kappa r) \cos (\ell \theta),
$$

and where $w^{n, m}$ is a fully identified function lifting the nonhomogeneous Neumann data. We shall intensively make use of the following expansions of the Hankel functions (cf., e.g., [35]):

$$
\begin{aligned}
& H_{m}^{(1)}(z)=\frac{1}{i \pi}(z / 2)^{-m} P_{m-1}\left((z / 2)^{2}\right) \\
& \quad+\left(1+\frac{2 i}{\pi} \ln (z / 2)\right)(z / 2)^{m} j_{m}\left((z / 2)^{2}\right)+\frac{1}{i \pi}(z / 2)^{m} y_{m}\left((z / 2)^{2}\right),
\end{aligned}
$$

where $P_{m-1}(\zeta)$ is a polynomial of degree $m-1$ (this term is discarded for $m=0$ ),

$$
P_{m-1}(\zeta)=\sum_{n=0}^{m-1} \frac{(m-1-n) !}{n !} \zeta^{n},
$$


and $j_{m}(\zeta)$ and $y_{m}(\zeta)$ are two analytical functions on the complex plane $\mathbb{C}$ given by

$$
\begin{aligned}
& j_{m}(\zeta)=\sum_{n=0}^{\infty} \frac{(-1)^{n}}{n !} \frac{1}{(m+n) !} \zeta^{n}, \\
& y_{m}(\zeta)=\sum_{n=0}^{\infty}(\psi(m+n+1)+\psi(n+1)) \frac{(-1)^{n}}{n !(m+n) !} \zeta^{n} .
\end{aligned}
$$

These three functions satisfy

$$
P_{m-1}(0)=(m-1) !, \quad j_{m}(0)=\frac{1}{m !}, \quad y_{m}(0)=\frac{\psi(m+1)-\gamma}{m !},
$$

where $\psi$ is the logarithmic derivative of the function $\Gamma$,

$$
\psi(z)=\Gamma^{\prime}(z) / \Gamma(z) .
$$

In particular, we directly get from the above expression of $H_{m}^{(1)}(z)$ their behavior for the small values of the argument

$$
\begin{gathered}
H_{0}^{(1)}(z)=1+\frac{2 i}{\pi}(\ln z / 2+\gamma)+O\left(|z|^{2} \ln |z|\right), \\
H_{1}^{(1)}(z)=-\frac{2 i}{\pi} \frac{1}{z}+O(|z| \ln |z|)
\end{gathered}
$$

and more generally for $m=2,3, \ldots$,

$$
H_{m}^{(1)}(z)=\frac{2^{m}(m-1) !}{i \pi} \frac{1}{z^{m}}+o\left(|z|^{-m}\right) .
$$

2.4. Characterization of the solutions to the inner problems.

2.4.1. The inner problems. In some meaning contrary to the outer coefficients, the inner ones $\Pi^{n, m}$ keep the $\mathrm{H}^{1}$ regularity of the exact solution $u^{\delta}$ except at infinity where their behavior cannot be restrained a priori. Thus, the adequate functional framework is given this once by the following Fréchet space:

$$
\mathrm{H}_{\text {loc }}^{1}(\widehat{\widehat{\Omega}})=\left\{\Pi \in \mathcal{D}^{\prime}(\widehat{\Omega}): \varphi \Pi \in \mathrm{H}^{1}(\widehat{\Omega}), \forall \varphi \in \mathcal{D}\left(\mathbb{R}^{2}\right)\right\} .
$$

Plugging expansion (2.11) into (1.10), we readily get the following systems:

$$
\begin{cases}\Pi^{n, m} \in \mathrm{H}_{\mathrm{loc}}^{1}(\bar{\Omega}), & \\ \nabla_{X, Y} \cdot \frac{1}{\varepsilon} \nabla_{X, Y} \Pi^{n, m}=-\kappa^{2} \mu \Pi^{n-2, m} & \text { in } \widehat{\Omega}, \\ \frac{1}{\varepsilon} \partial_{Y} \Pi^{n, m}=0 & \text { on } \partial \widehat{\Omega}\end{cases}
$$

where as above $1 / \varepsilon$ and $\mu$ denote functions equal to the reciprocal of the dielectric permittivity and the magnetic permeability of the substrate respectively for $Y<0$ and are equal to 1 for $Y>0$.

The inner asymptotic expansions for $n \leq 2$ make use only of the solutions to the homogeneous problem associated to (2.37):

$$
\begin{cases}\Pi \in \mathrm{H}_{\mathrm{loc}}^{1}(\widehat{\widehat{\Omega}}), & \\ \nabla_{X, Y} \cdot \frac{1}{\varepsilon} \nabla_{X, Y} \Pi=0 & \text { in } \widehat{\Omega}, \\ \frac{1}{\varepsilon} \partial_{Y} \Pi=0 & \text { on } \partial \widehat{\Omega},\end{cases}
$$


which behave as follows for $Y>0$ :

$$
\Pi(X, Y)=a+b \ln R+\underset{R \rightarrow \infty}{o}(1)
$$

and are not increasing at an exponential rate at the back of the cavity. We denote by $(R, \theta)$ the polar coordinates associated to the fast variables $(X, Y)$ since it is possible here to identify the fast and the slow angular variables.

Obviously inner problem (2.38) corresponds to the behavior of the field near the edge of the patch, which is static by nature. Previous works have already been devoted to such kinds of problems [21, 7, 6. However, all these references addressed the capacitance problem where the field is characterized from Dirichlet data on the boundary. It does not seem that the same techniques can be adapted to the present context since the static field has now to be determined from its asymptotic behavior at infinity.

It is instructive first to examine the case where the dielectric constants $\varepsilon$ and $\mu$ are 1 , which moreover constitutes the setting where the error bounds are proved. The results are embodied in the following proposition.

Proposition 2.3. For $\varepsilon=1$, every solution to (2.38) is in the form

$$
\Pi(X, Y)=\sum_{-\infty}^{-1} a_{n} \Re \omega^{n}(X, Y)+a+b \Re \ln \omega(X, Y)+\sum_{1}^{+\infty} a_{n} \Re \omega^{n}(X, Y),
$$

where $(X, Y) \rightarrow \omega(X, Y)$ is the conformal transform adapted from 27] which maps the lower complex half-plane

$$
\{\omega \in \mathbb{C}: \Im \omega<0\}
$$

onto $\widehat{\Omega}$ and which is implicitly defined through the following relation:

$$
Z=1-\omega(X, Y)+\ln \omega(X, Y), Z=X+i Y .
$$

Moreover the constants $a_{n}$ for $n \leq-1$ are all zero if and only if $\Pi(X, Y)$ does not increase at an exponential rate as $X \rightarrow-\infty$ inside the cavity and the constants $a_{n}$ for $n \geq 1$ are all zero if $\Pi$ has at most a logarithmic growth as $R \rightarrow \infty$ for $Y>0$. The function $(X, Y) \rightarrow \Re \ln \omega(X, Y)$ behaves as follows:

$$
\begin{aligned}
& \Re \ln \omega(X, Y)=\ln R-\frac{\ln R \cos \Phi-(\pi-\Phi) \sin \Phi}{R}-\frac{\cos \Phi}{R} \\
& +\underset{R \rightarrow \infty}{O}\left(\frac{\ln ^{2} R}{R^{2}}\right) \text { for } Y>0, \\
& \Re \ln \omega(X, Y)=\ln X-\frac{1+\ln X}{X}+\underset{X \rightarrow \infty}{O}\left(\frac{\ln ^{2} X}{X^{2}}\right) \text { for }-\pi<Y<0, \\
& \Re \ln \omega(X, Y)=X-1+\underset{X \rightarrow-\infty}{o}\left(|X|^{-m}\right) \text { for all } m .
\end{aligned}
$$

Proof. Expansion (2.40) is simply obtained by a separation of variables after noting that the variable change $(X, Y) \rightarrow \omega(X, Y)$ gives rise to a smooth function near the point 
$1=\omega(0,0)$. The rest of the proof follows from the following elementary estimates:

$$
\left\{\begin{array}{l}
\Re \ln \omega(X, Y)=\ln R+\underset{R \rightarrow \infty}{o}(1), \\
\Re \omega^{n}(X, Y)=(-1)^{n} R^{n} \cos n \theta+\underset{R \rightarrow \infty}{o}\left(R^{n}\right)(n=1,2, \ldots), \\
\ln \omega(X, Y)=(X-1+i Y)+\underset{X \rightarrow-\infty}{o}\left(|X|^{-m}\right) \text { for all } m,-\pi<Y<0, \\
\omega(X, Y)=-Z+\ln (-Z)+1-\frac{\ln (-Z)+1}{Z}+\underset{R \rightarrow \infty}{O}\left(\frac{\ln ^{2} R}{R^{2}}\right), \\
\ln \omega(X, Y)=\ln (-Z)-\frac{(\ln (-Z)+1)}{Z}+\underset{R \rightarrow \infty}{O}\left(\frac{\ln ^{2} R}{R^{2}}\right) .
\end{array}\right.
$$

Our goal is now to extend this result to the case of a substrate with general dielectric constants $\varepsilon$ and $\mu$ and mainly to build a function $\Pi$ playing the same role as the above function $\Re \ln \omega$. This requires some preparatory lemmas.

Lemma 2.4. The space $\mathrm{V}$ of $\Theta \in H_{\mathrm{loc}}^{1}(\overline{\widehat{\Omega}})$ such that all of the following functions $\left.\frac{1}{1+R \ln R} \Theta\right|_{Y>0},\left.\frac{1}{1+R \ln R} \Theta\right|_{Y>0}$, and $\left.\frac{1}{1+X} \Theta\right|_{-\pi<Y<0}$ are $L^{2}$ is a Hilbert space of BeppoLevi type which contains the constants. For any $T \in \mathcal{D}^{\prime}(\widehat{\Omega})$ which can be extended as a continuous linear form on $\mathrm{V}$, the problem

$$
\begin{cases}\Theta \in \mathrm{V}, & \\ -\nabla_{X, Y} \cdot \frac{1}{\varepsilon} \nabla_{X, Y} \Theta=T & \text { in } \widehat{\Omega}, \\ \frac{1}{\varepsilon} \partial_{Y} \Theta=0 & \text { on } \partial \widehat{\Omega}\end{cases}
$$

admits a solution if and only if

$$
\langle T, 1\rangle_{\mathrm{V}^{\prime}, \mathrm{V}}=0
$$

and this solution is unique up to an additive constant.

Proof. It is obtained by a straightforward application of the variational method using the coerciveness results stated in [30].

Borrowing the technique for constructing a solution to (2.38) behaving as $\ln R$ as $R \rightarrow \infty$ from [2], we consider the "profil" $\mathrm{P}_{\alpha}$ defined almost everywhere on $\widehat{\Omega}$ for any given complex constant $\alpha$ by

$$
\mathrm{P}_{\alpha}(X, Y)= \begin{cases}\ln R, & \text { if } Y>0, \\ \ln X, & \text { if } X>0 \text { and }-\pi<Y<0, \\ \alpha X, & \text { if } X<0 \text { and }-\pi<Y<0 .\end{cases}
$$

We then introduce the cutoff function

$$
\Xi=1-\chi \otimes X, Y \chi
$$

with $\chi \in \mathcal{D}(\mathbb{R})$ such that $\chi \geq 0, \chi(t)=1$ for $|t| \leq \pi$ and $\chi(t)=0$ for $|t| \geq 2 \pi$. The function

$$
T_{\alpha}=\nabla_{X, Y} \cdot \frac{1}{\varepsilon} \nabla_{X, Y}\left(\Xi \mathrm{P}_{\alpha}\right)
$$

is in $L^{\infty}(\widehat{\Omega})$ and has a bounded support which does not intersect the opening of the cavity. The introduction of the multiplicative constant $\alpha$ is justified by the property stated in the following lemma. 
Lemma 2.5. The function $T_{\alpha}$ defined in (2.50) is such that

$$
\int_{\widehat{\Omega}} T_{\alpha} d X d Y=\pi\left(1-\frac{\alpha}{\varepsilon}\right)
$$

Proof. For $L$ sufficiently large, we have by simple integrations in $X$ and the Green formula,

$$
\begin{aligned}
\frac{\pi \alpha}{\varepsilon} \int_{-L}^{0} \partial_{X}^{2}(1-\chi(X)) X d X+ & \frac{\pi}{\varepsilon} \int_{0}^{L} \partial_{X}^{2}(1-\chi(X)) \ln X d X \\
& +\int_{\{R<L, Y>0\}} \Delta(\Xi \ln R) d X d Y=-\frac{\pi \alpha}{\varepsilon}+\frac{\pi}{\varepsilon L}+\pi .
\end{aligned}
$$

The result is obtained by noting that the left-hand side of the above relation is equal to the integral of $T_{\alpha}$ on the strip $\{-\pi<Y<0, X<L\}$ and the half-disk $\{R<L, Y>0\}$ and letting $L$ tend to $+\infty$.

We can hence use Lemma 2.4 to get a solution $\Theta_{\varepsilon} \in \mathrm{V}$ to problem (2.46) with $T=T_{\varepsilon}$. This enables us to define a solution to (2.38),

$$
\Pi_{C}=\Xi \mathrm{P}_{\varepsilon}+\Theta_{\varepsilon}+C,
$$

depending on an additive constant $C$ which is chosen as the following lemma indicates it.

Lemma 2.6. The above additive constant $C$ can be chosen such that the function $\Pi_{C}$, then denoted by $\Pi^{\varepsilon}$, has the following asymptotic behaviors:

$$
\left\{\begin{array}{l}
\Pi^{\varepsilon}=\ln R+\underset{R \rightarrow \infty}{o}(1) \text { for } Y>0, \\
\Pi^{\varepsilon}=\ln X+\underset{X \rightarrow+\infty}{o}(1) \text { for }-\pi<Y<0, \\
\Pi^{\varepsilon}=\varepsilon X-\xi_{\varepsilon}+\underset{X \rightarrow-\infty}{o}\left(|X|^{-m}\right) \text { for }-\pi<Y<0(\forall m \geq 1)
\end{array}\right.
$$

with $\xi_{1}=1$.

Proof. Using a technique of proof introduced in [2, we consider the Mellin transform to reduce the asymptotic behavior of $\Xi \Pi_{C}$ to meromorphic properties of

$$
\begin{gathered}
\Lambda(\lambda, \theta)=\left(\left.\Xi \Pi_{C}\right|_{Y>0}\right)^{\wedge}(\lambda, \theta)=\int_{0}^{+\infty} R^{-\lambda-1}\left(\Xi \Pi_{C}\right)(R, \theta) d R, \\
L(\lambda, \theta)=\left(\left.\Xi \Pi_{C}\right|_{X>0,-\pi<Y<0}\right) \hat{(\lambda, \theta)} \int_{0}^{+\infty} X^{-\lambda-1}(1-\chi(X)) \Pi_{C}(X, Y) d X .
\end{gathered}
$$

The above functions are well-defined and holomorphic in $\lambda$ in the half-plane $\Re \lambda>0$. This results from the elementary properties of the Mellin transform (see for example [10, 29]) that $\Lambda$ and $L$ satisfy the following equations, which are conveniently grouped as follows:

$$
\begin{aligned}
& \left\{\begin{array}{l}
\left(\partial_{\theta}^{2}+\lambda^{2}\right) \Lambda=F \\
\left.\partial_{\theta} \Lambda\right|_{\theta=\pi}=0,\left.\partial_{\theta} \Lambda\right|_{\theta=0}=\frac{1}{\varepsilon} \partial_{Y}\left(\left.X \Xi \Pi_{C}\right|_{Y=0, X>0}\right)^{\wedge}=\left.\frac{1}{\varepsilon}\left(\partial_{Y} \tau_{-1}^{\lambda} L\right)\right|_{Y=0},
\end{array}\right. \\
& \left\{\begin{array}{l}
\partial_{Y}^{2} L=G-(\lambda+1)(\lambda+2) \tau_{2}^{\lambda} \Lambda \\
\left.\partial_{Y} L\right|_{Y=-\pi}=0,\left.L\right|_{Y=0}=\left.\Lambda\right|_{\theta=0}
\end{array}\right.
\end{aligned}
$$


where $(\lambda, \theta) \rightarrow F(\lambda, \theta)$ and $(\lambda, Y) \rightarrow G(\lambda, Y)$ are holomorphic functions of $\lambda$ on all of the complex plane, and $\tau_{p}^{\lambda} \Lambda$ is the translation operator defined by $\tau_{p}^{\lambda} \Lambda(\lambda, \cdot)=\Lambda(\lambda+p, \cdot)$. An elementary calculation yields

$$
\begin{aligned}
\Lambda(\lambda, \theta)= & A \cos \lambda \theta+B \frac{\sin \lambda \theta}{\lambda}+\int_{0}^{\theta} \frac{\sin \lambda(\theta-s)}{\lambda} F(\lambda, s) d s \\
L(\lambda, \theta)=A+ & Y \int_{-\pi}^{0}\left(G-(\lambda+1)(\lambda+2) \tau_{2}^{\lambda} \Lambda\right) d s \\
& +\int_{0}^{Y}(Y-s)\left(G-(\lambda+1)(\lambda+2) \tau_{2}^{\lambda} \Lambda\right) d s
\end{aligned}
$$

with

$$
\begin{gathered}
B=\frac{1}{\varepsilon} \tau_{-1}^{\lambda} \int_{-\pi}^{0} G(\lambda, s) d s-\frac{1}{\varepsilon} \lambda(\lambda+1) \tau_{1}^{\lambda} \int_{-\pi}^{0} L(\lambda, s) d s \\
A=B \frac{\cos \lambda \pi}{\lambda \sin \lambda \pi}+\frac{1}{\lambda \sin \lambda \pi} \int_{0}^{\pi} \cos \lambda(\pi-s) F(\lambda, s) d s .
\end{gathered}
$$

The functions $A, B, \Lambda$ and $L$ can be extended as meromorphic functions of $\lambda$ on $\Re \lambda>-1$ with a pole of order 2 at 0 as follows:

$$
\begin{aligned}
& A=\frac{A_{1}^{(0)}}{\lambda^{2}}+\frac{A_{0}^{(0)}}{\lambda}+A_{r}^{(0)}, B=B_{r}^{(0)}, \\
& \Lambda(\lambda, \theta)=\frac{A_{1}^{(0)}}{\lambda^{2}}+\frac{A_{0}^{(0)}}{\lambda}+\Lambda_{r}^{(0)}(\lambda, \theta), \\
& L(\lambda, Y)=\frac{A_{1}^{(0)}}{\lambda^{2}}+\frac{A_{0}^{(0)}}{\lambda}+L_{r}^{(0)}(\lambda, \theta),
\end{aligned}
$$

where the subscript $r$ indicates that the corresponding function is holomorphic for $\Re \lambda>$ -1 . The link between the holomorphic properties of the Mellin transform of a function and its behavior at infinity directly yields that

$$
\left\{\begin{array}{l}
\Pi_{C}(R, \theta)=A_{1}^{(0)} \ln R+A_{0}^{(0)}+\underset{R \rightarrow \infty}{o}(1) \text { for } Y>0, \\
\Pi_{C}(X, Y)=A_{1}^{(0)} \ln X+A_{0}^{(0)}+\underset{X \rightarrow \infty}{o}(1) \text { for }-\pi<Y<0 .
\end{array}\right.
$$

Noting now that if the constant $C$ is shifted to $C+C^{\prime}$, the related functions $\Lambda^{C^{\prime}}$ and $L^{C^{\prime}}$ are linked to $\Lambda$ and $L$ respectively by

$$
\Lambda^{C^{\prime}}=\Lambda+\frac{C^{\prime}}{\lambda}+\Lambda_{r}^{C^{\prime}}, L^{C^{\prime}}=L+\frac{C^{\prime}}{\lambda}+L_{r}^{C^{\prime}}
$$

with $\Lambda_{r}^{C^{\prime}}$ and $L_{r}^{C^{\prime}}$ two holomorphic functions on all of the complex plane. Such a shift then modifies only the coefficient $A_{0}^{(0)}$ with no change of the other poles or their coefficients. By then suitably choosing the constant $C$, we can assume that $A_{0}^{(0)}=0$. Noting then that a function which behaves as $\ln R$ as $R \rightarrow \infty$ cannot belong to the variational space $\mathrm{V}$, we readily get that $A_{1}^{(0)}=1$. The asymptotic behavior in the cavity is obtained more easily by expanding $\Pi^{\varepsilon}$ from a usual separation of variables and making use once more of the fact that the part $\varepsilon X$ of $\Pi^{\varepsilon}$ increasing in modulus as $X \rightarrow-\infty$ is not in V. The value of the constant $\xi_{\varepsilon}$ for $\varepsilon=1$ is obtained from the expression $\Pi^{1}=\Re \ln \omega$ obtained for this function for $\varepsilon=1$. 
We are now in position to extend the above proposition established for $\varepsilon=\mu=1$ to a general substrate.

Proposition 2.7. For a general $\varepsilon$, every solution $\Pi$ to (2.38) which does not grow at an exponential rate as $X \rightarrow-\infty$ inside the cavity and increases at most as $\ln R$ for $R \rightarrow \infty$ and $Y>0$ can be expressed in terms of two constants $a$ and $b$ as follows:

$$
\Pi=a+b \Pi^{\varepsilon} .
$$

Its asymptotic behavior at infinity in the various zones of $\widehat{\Omega}$ can be expressed from that of $\Pi^{\varepsilon}$ :

$$
\begin{gathered}
\Pi^{\varepsilon}=\ln R-\frac{1}{\varepsilon}\left(\frac{\ln R \cos \theta-(\pi-\theta) \sin \theta}{R}+\eta_{\varepsilon} \frac{\cos \theta}{R}\right)+\underset{R \rightarrow \infty}{o}\left(R^{-1}\right) \text { for } Y>0, \\
\Pi^{\varepsilon}=\ln X-\frac{1}{\varepsilon}\left(\frac{\ln X+\eta_{\varepsilon}}{X}\right)+\underset{X \rightarrow \infty}{o}\left(X^{-1}\right) \text { for }-\pi<Y<0,
\end{gathered}
$$

and for all positive integers $m$,

$$
\Pi^{\varepsilon}=\varepsilon X-\xi_{\varepsilon}+\underset{X \rightarrow-\infty}{o}\left(|X|^{-m}\right) \text { for }-\pi<Y<0 .
$$

The two constants $\eta_{\varepsilon}$ and $\xi_{\varepsilon}$ depend only on $\varepsilon$ and are equal to 1 for $\varepsilon=1$. An explicit value for $\xi_{\varepsilon}$ will be given below.

Proof. The proof is obtained by a simple determination of the poles at $\lambda=-1$ of the above functions $A, B, \Lambda$ and $L$, taking care of the contribution brought then by the poles at $\lambda=0$.

2.5. The matching procedure. According to the above discussion, the outer and the inner coefficients, respectively $u^{n, m}, u_{\mathrm{L}}^{n, m}, u_{\mathrm{C}}^{n, m}$ and $\Pi^{n, m}$, are defined up to some coefficients $C_{p}^{n, m}, R^{n, m}, a^{n, m}$ and $b^{n, m}$. In this section, we show how they can be determined using a matching procedure close to the so-called Van Dyke matching principle [9, 13, 12. This is done recursively through the following steps at each order $N$.

Step 1. The inner asymptotic expansions in the half-plane and in the cavity are truncated at order $N$ :

$$
\begin{aligned}
& u_{\delta}^{N}(x, y)=\sum_{n=0 m=0}^{N} \sum^{n} \delta^{n} \ln ^{m} \delta u^{n, m}(x, y), \\
& u_{\mathrm{C}, \delta}^{N}(x, Y)=\sum_{n=0}^{N} \sum_{m=0}^{n} \delta^{n} \ln ^{m} \delta u_{\mathrm{C}}^{n, m}(x, Y) .
\end{aligned}
$$

They are next expressed with respect to the $(X, Y)$ variables and expanded at order $N$ (any term that goes to 0 faster than $\delta^{N}$ is discarded and joins the remainder):

$$
\begin{aligned}
& u_{\delta}^{N}(\delta X, \delta Y)=\sum_{n=0}^{N} \sum_{m=0}^{n} \delta^{n} \ln ^{m} \delta U_{N}^{n, m}(X, Y)+\delta^{N} \varepsilon_{\delta}, \\
& u_{\mathrm{C}, \delta}^{N}(\delta X, Y)=\sum_{n=0}^{N} \sum_{m=0}^{n} \delta^{n} \ln ^{m} \delta U_{\mathrm{C}}^{n, m}(X, Y)+\delta^{N} \varepsilon_{\delta} .
\end{aligned}
$$


The functions $U_{N}^{n, m}$ and $U_{\mathrm{C}}^{n, m}$ are independent of $\delta$. It is worth mentioning that the strong connection between $u^{n, m}$ and the layer coefficients $u_{\mathrm{L}}^{n, m}$ avoids having to match the inner expansion in the substrate with the outer one.

Step 2. The matching conditions are then obtained from the following identifications:

$$
\begin{aligned}
& \Pi^{n, m}(X, Y)=U_{N}^{n, m}(X, Y)+\underset{R \rightarrow \infty}{o}\left(R^{n-N}\right), \\
& \Pi^{n, m}(X, Y)=U_{\mathrm{C}}^{n, m}(X, Y)+\underset{X \rightarrow-\infty}{o}\left(|X|^{n-N}\right), \quad 0 \leq m \leq n \leq N .
\end{aligned}
$$

REMARK 2.8. The matching conditions (2.73) generally yield an overdetermined system for the unknowns $C_{\ell}^{n, m}, a^{n, m}, b^{n, m}$, and $R^{n, m}$. Some of these equations have hence to be satisfied as compatibility conditions strengthening the validity of the asymptotic expansion.

2.6. Determination of the asymptotic expansions. We limit the exposure to the main steps and ingredients yielding the asymptotic expansions up to order 2 skipping some long but straightforward calculations (see [22] for the details).

2.6.1. Zeroth-order asymptotic expansions. From (2.33) and (2.35), we respectively get

$$
H_{0}^{(1)}(\kappa \delta R)=\frac{2 i}{\pi}(\ln \delta+\ln R)+\gamma_{\kappa}+O\left(\delta^{2} \ln \delta\right),
$$

where $\gamma_{\kappa}$ is the constant defined by

$$
\gamma_{\kappa}=1+\frac{2 i}{\pi}\left(\gamma+\ln \frac{\kappa}{2}\right)
$$

and

$$
H_{m}^{(1)}(\kappa \delta R)=\frac{1}{\delta^{m}} \frac{2^{m}(m-1) !}{i \pi \kappa^{m}} \frac{1}{R^{m}}+o\left(\delta^{-m}\right) \text { for } m=1,2, \ldots
$$

Particularizing (2.24) and (2.25) to $u^{0,0}$, we thus obtain that the coefficients $C_{\ell}^{0,0}$ are all equal to zero; otherwise, $u_{\delta}^{0}(\delta X, \delta Y)$ will involve terms in $\delta^{-p} \ln ^{q} \delta$, with $p, q \geq 0$ and $p+q>0$, which cannot be matched with the inner expansion. As a result, we get

$$
u^{0,0}=0, u_{\mathrm{L}}^{0,0}=0, \Pi^{0,0}=0 .
$$

Now from (2.21), we can write

$$
u_{\mathrm{C}}^{0,0}(\delta X, Y)=\exp \left(i \kappa_{\mathrm{S}} \delta X\right)+R^{0,0} \exp \left(-i \kappa_{\mathrm{S}} \delta X\right)=1+R^{0,0}+\underset{\delta \rightarrow 0}{o}(1) .
$$

The matching conditions (2.73) hence yield $R^{0,0}=-1$ and thus

$$
u_{\mathrm{C}}^{0,0}(x, Y)=2 i \sin \kappa_{\mathrm{S}} x .
$$

REMARK 2.9. Property $R^{0,0}=-1$ is equivalent to the following condition:

$$
u_{\mathrm{C}}^{0,0}(0, Y)=0,-\pi<Y<0,
$$

set on the open side of the cavity. In this way, we recover the empirically assumed magnetic wall condition, which is a basic step in the derivation of the cavity model (cf., e.g., [1, 3]). 
2.6.2. First-order expansions. Since $u_{\mathrm{L}}^{0,0}=0$, proceeding as for $u^{0,0}$ we get that $u^{1,1}=$ 0 and $u^{1,0}(x, y)=C_{0}^{10} H_{0}^{(1)}(\kappa r)$. Using (2.74), we can then write

$$
u_{\delta}^{1}(\delta X, \delta Y)=\delta C_{0}^{1,0}\left(\frac{2 i}{\pi} \ln R+\gamma_{\kappa}\right)+\delta \ln \delta C_{0}^{1,0} \frac{2 i}{\pi}+o(\delta) .
$$

Matching conditions (2.73) and the general form (2.67) of the solutions to the homogeneous inner problems growing at most as $\ln R$ as $R \rightarrow \infty$ for $Y>0$ then yields

$$
\left\{\begin{array}{l}
\Pi^{1,0}(X, Y)=a^{1,0}+b^{1,0} \Pi^{\varepsilon} \\
\Pi^{1,1}(X, Y)=a^{1,1}+b^{1,1} \Pi^{\varepsilon}
\end{array}\right.
$$

with

$$
\begin{cases}a^{1,0} / C_{0}^{1,0}=\gamma_{\kappa}, & b^{1,0} / C_{0}^{1,0}=2 i / \pi \\ a^{1,1} / C_{0}^{1,0}=2 i / \pi, & b^{1,1}=0 .\end{cases}
$$

To complete the determination of the expansions, we now express the outer expansion inside the cavity in terms of the fast variables and expand it in $\delta$ :

$$
\begin{aligned}
u_{\mathrm{C}, \delta}^{1}(\delta X, Y) & =2 i \sin \kappa_{\mathrm{s}} \delta X+\delta\left(R^{1,0}+\ln \delta R^{1,1}\right) \exp (-i \kappa \delta X) \\
& =\delta\left(2 i \kappa_{\mathrm{s}} X+R^{1,0}\right)+\delta \ln \delta R^{1,1}+o(\delta)
\end{aligned}
$$

to get

$$
\left\{\begin{array}{l}
U_{\mathrm{C}}^{1,0}(X, Y)=2 i \kappa_{\mathrm{S}} X+R^{1,0} \\
U_{\mathrm{C}}^{1,1}(X, Y)=R^{1,1}
\end{array}\right.
$$

The matching rules (2.73) and the asymptotic behavior of $\Pi^{\varepsilon}$ (cf. (2.70) $)$ when $(X, Y)$ goes to infinity inside the cavity then give

$$
\left\{\begin{array}{l}
b^{1,0}\left(\varepsilon X-\xi_{\varepsilon}\right)+a^{1,0}=2 i \kappa_{\mathrm{S}} X+R^{1,0}+o\left(|X|^{-1}\right) \\
b^{1,1}\left(\varepsilon X-\xi_{\varepsilon}\right)+a^{1,1}=R^{1,1}+o\left(|X|^{-1}\right)
\end{array}\right.
$$

This yields

$$
C_{0}^{1,0}=\pi \kappa_{\mathrm{S}} / \varepsilon=\pi \kappa \zeta_{\mathrm{s}}
$$

resulting in an expression in $\Omega$ for $u^{\delta}=\delta C_{0}^{1,0} H_{0}+o(\delta)$ in agreement with that found above from the Wiener-Hopf solution. Moreover, since

$$
\left\{\begin{array}{l}
R^{1,0}=a^{1,0}-b^{1,0} \xi_{\varepsilon}=C_{0}^{1,0}\left(\gamma_{\kappa}-\frac{2 i}{\pi} \xi_{\varepsilon}\right)=\pi \kappa \zeta_{\mathrm{s}}\left(\gamma_{\kappa}-\frac{2 i}{\pi} \xi_{\varepsilon}\right), \\
R^{1,1}=a^{1,1}-b^{1,1} \xi_{\varepsilon}=a^{1,1}=C_{0}^{1,0} \frac{2 i}{\pi}=2 i \kappa \zeta_{\mathrm{s}}
\end{array}\right.
$$

we can also deduce a first-order expansion of the reflection coefficient given by

$$
R^{\delta}=-1+\pi \kappa \zeta_{\mathrm{s}} \delta\left(1+\frac{2 i}{\pi}\left(\gamma+\ln \frac{\kappa \delta}{2}\right)-\frac{2 i}{\pi} \xi_{\varepsilon}\right)+o(\delta),
$$

which also agrees with the expression obtained in (2.9) from the Wiener-Hopf procedure. Moreover, the availability of the latter approximation provides an explicit expression for the constant $\xi_{\varepsilon}$ :

$$
\xi_{\varepsilon}=1-2 \varepsilon Q_{0}\left(\frac{1-\varepsilon}{1+\varepsilon}\right)+(\varepsilon-1) \ln 2 \pi .
$$

Only one term in the expansion corresponding to the substrate for $x>0$ is nonzero and according to (2.20) is given by

$$
u_{\mathrm{L}}^{1,0}(x, Y)=C_{0}^{1,0} H_{0}^{(1)}(\kappa x), x>0,-\pi<Y<0 .
$$


REMARK 2.10. The above derivation establishes that the cavity model for patch antennas is nothing else than a two-scale asymptotic expansion of the actual radiated field. It is worth recalling the main steps involved in the derivation of this model. At first, the tangential component of the magnetic field is assumed to be zero a priori at the opening of the cavity. This makes it possible to easily determine the electric field inside the cavity. Actually, the solution of the resulting boundary-value problem is done in terms of a modal expansion which carries out the dimension reduction near the edge of the patch. This gives the electric field at the opening of the cavity, expressed by $\left.\partial_{x}\left(2 i \sin \kappa_{\mathrm{s}} x\right)\right|_{x=0}=2 i \kappa_{\mathrm{s}}$ up to a multiplicative constant here, and, as a result, the field $\delta C_{0}^{1,0} H_{0}^{(1)}(\kappa r)$ radiated by the antenna.

2.6.3. Second-order asymptotic expansions. All $u_{\mathrm{L}}^{n, m}$ with $n \leq 1$ are zero except $u_{\mathrm{L}}^{1,0}$. Accordingly in (2.25) all of the $w^{2, m}$ but $w^{2,0}$ are zero, which in view of (2.20) satisfies a homogeneous Neumann boundary condition for $x<0$ and

$$
\partial_{y} w^{2,0}(x, 0)=-C_{0}^{1,0} \frac{\pi}{\varepsilon}\left(\partial_{x}^{2}+\kappa_{\mathrm{s}}^{2}\right) H_{0}^{(1)}(\kappa x) .
$$

From elementary properties of the Bessel functions, the above Neumann boundary condition can be put in the following form:

$$
\partial_{y} w^{2,0}(x, 0)=-C_{0}^{1,0} \frac{\pi}{\varepsilon}\left(\frac{\kappa}{x} H_{1}^{(1)}(\kappa x)+\kappa^{2}\left(n_{\mathrm{s}}^{2}-1\right) H_{0}^{(1)}(\kappa x)\right) .
$$

The general expression of the solutions to the singular boundary-value problem (2.24) obtained by Makhlouf [22] then gives

$$
w^{2,0}=C_{0}^{1,0} \frac{2 i}{\pi \varepsilon}\left(\mathrm{s}+r^{2,0}\right)
$$

where $r^{2,0}$ is a function in $H^{3-t}(\Omega)$ for all $t>0$ and $\mathrm{s}$ is a function with a bounded support, indefinitely differentiable up to the boundary except at $(0,0)$, with the following expression in the proximity of $(0,0)$ :

$$
\begin{aligned}
& \mathbf{s}=\mathbf{s}_{-1}+\mathbf{s}_{1}, \\
& \mathbf{s}_{-1}(r, \theta)=-\frac{1}{r}(\ln r \cos \theta-(\pi-\theta) \sin \theta)
\end{aligned}
$$

and $\mathrm{s}_{1}=r P_{m}(\theta, \ln r), P_{m}(\theta, z)$ being a polynomial of degree $m$ in $z$ whose coefficients $a_{\ell}(\theta)$ are $\mathcal{C}^{\infty}$ functions of $\theta$. The function $(r, \theta) \rightarrow \mathbf{s}_{-1}(r, \theta)$ is exactly the same as the one expressed in the variables $(R, \theta)$ which was already involved above for the description of the asymptotic behavior of $\Pi^{\varepsilon}$ when $R \rightarrow \infty$.

The matching rules (2.73) and straightforward but relatively long calculations which are not reproduced here yield the second-order expansion. The various terms $u^{2, m}, u_{\mathrm{C}}^{2, m}$ and $\Pi^{2, m}$ of this expansion are defined from (2.25), (2.21), and (2.67), respectively, in which the nonzero coefficients are given in Table 1 . 


\begin{tabular}{|c|c|c|c|}
\hline$(n, m)$ & $(2,0)$ & $(2,1)$ & $(2,2)$ \\
\hline$C_{0}^{n, m} / C_{0}^{1,0}$ & $-\pi \kappa \zeta_{\mathrm{s}}\left(\gamma_{\kappa}-\frac{2 i}{\pi} \xi_{\varepsilon}\right) / 2$ & $-i \kappa \zeta_{\mathrm{s}}$ & 0 \\
\hline$C_{1}^{n, m} / C_{0}^{1,0}$ & $\eta_{\varepsilon} \kappa / \varepsilon$ & $-\kappa / \varepsilon$ & 0 \\
\hline$a^{n, m} / C_{0}^{1,0}$ & $\begin{array}{c}\frac{2 i}{\pi \varepsilon} r^{2,0}(0,0) \\
-\frac{\pi \kappa \zeta_{\mathrm{s}}}{2} \gamma_{\kappa}\left(\gamma_{\kappa}-\frac{2 i}{\pi} \xi_{\varepsilon}\right)\end{array}$ & $-2 \kappa \zeta_{\mathrm{s}}\left(i \gamma_{\kappa}+\frac{\xi_{\varepsilon}}{\pi}\right)$ & $2 \kappa \zeta_{\mathrm{s}} / \pi$ \\
\hline$b^{n, m} / C_{0}^{1,0}$ & $-i \kappa \zeta_{\mathrm{s}}\left(\gamma_{\kappa}-\frac{2 i}{\pi} \xi_{\varepsilon}\right)$ & $2 \kappa \zeta_{\mathrm{s}} / \pi$ & 0 \\
\hline $\begin{array}{l}R^{n, m} / C_{0}^{1,0}= \\
\quad\left(a^{n, m}-\xi_{\varepsilon} b^{n, m}\right) / C_{0}^{1,0}\end{array}$ & $\begin{array}{c}\frac{2 i}{\pi \varepsilon} r^{2,0}(0,0) \\
-\frac{\pi \kappa \zeta_{\mathrm{s}}}{2}\left(\gamma_{\kappa}-\frac{2 i}{\pi} \xi_{\varepsilon}\right)^{2}\end{array}$ & $-2 \kappa \zeta_{\mathrm{s}}\left(i \gamma_{\kappa}+\frac{2 \xi_{\varepsilon}}{\pi}\right)$ & $2 \kappa \zeta_{\mathrm{s}} / \pi$ \\
\hline
\end{tabular}

Let us just mention the functions involved in the matching procedure:

$$
\begin{aligned}
& \left\{\begin{array}{l}
U_{2}^{0,0}(X, Y)=0, \\
U_{2}^{1,0}(X, Y)=C_{0}^{1,0}\left(\frac{2 i}{\pi} \ln R+\gamma_{\kappa}-\frac{2 i}{\pi \varepsilon} \frac{\ln r \cos \theta-(\pi-\theta) \sin \theta}{R}\right)-C_{1}^{2,0} \frac{2 i}{\pi \kappa} \frac{\cos \theta}{R}, \\
U_{2}^{1,1}(X, Y)=\frac{2 i}{\pi} C_{0}^{1,0}-\frac{2 i}{\pi}\left(\frac{1}{\varepsilon} C_{0}^{1,0}+\frac{1}{\kappa} C_{1}^{2,1}\right) \frac{\cos \theta}{R}, \\
U_{2}^{2,0}(X, Y)=\frac{2 i}{\pi} C_{0}^{2,0} \ln R+C_{0}^{2,0} \gamma_{\kappa}+\frac{2 i}{\pi} C_{0}^{1,0} r^{2,0}(0,0), \\
U_{2}^{2,1}(X, Y)=\frac{2 i}{\pi} C_{0}^{2,1} \ln R+C_{0}^{2,1} \gamma_{\kappa}+\frac{2 i}{\pi} C_{0}^{2,0}, \\
U_{2}^{2,2}(X, Y)=\frac{2 i}{\pi} C_{0}^{2,1},
\end{array}\right. \\
& \left\{\begin{array}{l}
U_{\mathrm{C}}^{2,0}(X, Y)=-i \kappa_{\mathrm{s}} R^{1,0} X+R^{2,0}, \\
U_{\mathrm{C}}^{2,1}(X, Y)=-i \kappa_{\mathrm{S}} R^{1,1} X+R^{2,1}, \\
U_{\mathrm{C}}^{2,2}(X, Y)=R^{2,2} .
\end{array}\right.
\end{aligned}
$$

A quite remarkable feature of this expansion lies in the connection between the singularities of $u^{2,0}$, the solution of an instance of problem (2.24) and the lower-order terms in the asymptotic expansion of $\Pi^{\varepsilon}$ as $R \rightarrow \infty$.

Finally, since $u^{0,0}=0$, the coefficients related to the layer are simply given by

$$
u_{\mathrm{L}}^{n, m}(x, Y)=u^{n, m}(x, 0)(0 \leq m \leq n \leq 2) .
$$

3. Error estimates. In the previous section, we built a second-order asymptotic expansion of the solution of problem (1.10). This section is dedicated to proving error estimates that give a rigorous justification to this formal asymptotic expansion. To avoid too involved nonexplicit estimates based on the Mellin transform, we limit ourselves here to the case of a substrate with $\varepsilon=\mu=1$. It is clear however that the same estimates hold true simply by replacing the semi-explicit function $\Re \ln \omega$ by $\Pi^{\varepsilon}$, which was implicitly defined above as the solution of a particular boundary-value problem.

3.1. The uniformly valid approximation. Neither the outer nor the inner asymptotic expansions can be used to approximate $u^{\delta}$ everywhere. A uniformly valid approximation can be built by suitably mixing both of them. Following the approach described in [9, we do this by considering a suitable partition of unity, which can be described as follows. Let $\chi$ be the cutoff function defined just after (2.49). From its scaled version 
$\chi_{\delta}(x)=\chi(x / \sqrt{\delta})$, we consider the cutoff function of the plane $\Phi_{\delta}$ given by

$$
\Phi_{\delta}=\chi_{\delta} \otimes_{x, y} \chi_{\delta} .
$$

A uniformly valid approximation $u^{2, \delta}$ can then be defined by

$$
u_{\mathrm{uv}}^{2, \delta}=\Phi_{\delta} u_{\mathrm{inn}}^{2, \delta}+\left(1-\Phi_{\delta}\right) u_{\mathrm{out}}^{2, \delta},
$$

where

$$
\begin{aligned}
& u_{\text {out }}^{2, \delta}(x, y)= \begin{cases}\sum_{0 \leq m \leq n \leq 2} \delta^{n} \ln ^{m} \delta u^{n, m}(x, y), & \text { if }(x, y) \in \Omega, \\
\sum_{0 \leq m \leq n \leq 2} \delta^{n} \ln ^{m} \delta u_{\mathrm{L}}^{n, m}(x, y / \delta), & \text { if }(x, y / \delta) \in \widehat{\Omega}_{\mathrm{L}}, \\
\sum_{0 \leq m \leq n \leq 2} \delta^{n} \ln ^{m} \delta u_{\mathrm{C}}^{n, m}(x, y / \delta), & \text { if }(x, y / \delta) \in \widehat{\Omega}_{\mathrm{C}},\end{cases} \\
& u_{\text {inn }}^{2, \delta}(x, y)=\sum_{0 \leq m \leq n \leq 2} \delta^{n} \ln ^{m}(\delta) \widetilde{\Pi}^{n, m}(x, y) \text { for }(x, y) \in \Omega^{\delta},
\end{aligned}
$$

and $\widetilde{\Pi}^{n, m}$ is the function defined on $\Omega^{\delta}$ by

$$
\widetilde{\Pi}^{n, m}(x, y)=\Pi^{n, m}(x / \delta, y / \delta) .
$$

REMARK 3.1. The uniformly valid approximation is equal to the outer expansion away from the opening of the cavity and to the inner expansion in an "asymptotic" neighborhood of this opening. More precisely, we have

$$
u_{\mathrm{uv}}^{2, \delta}=u_{\text {out }}^{2, \delta} \text { for } \max (|x|,|y|) \geq 2 \sqrt{\delta} \quad \text { and } \quad u_{\mathrm{uv}}^{2, \delta}=u_{\text {inn }}^{2, \delta} \text { for } \max (|x|,|y|) \leq \sqrt{\delta} .
$$

It is only in the matching zone

$$
C_{\delta}^{\infty}=\left\{(x, y) \in \Omega_{\delta}: \sqrt{\delta} \leq|x|,|y| \leq 2 \sqrt{\delta}\right\}
$$

that both terms in formula (3.2) are not zero This is rather natural since either the inner or the outer expansion can be used to approximate $u^{\delta}$ there.

A quite natural manner for getting a bound on the error resulting from the approximation of $u^{\delta}$ by $u_{\mathrm{uv}}^{2, \delta}$ is to consider a consistency-stability approach. Starting from the stability result (1.14), the error bound is then reduced to an estimate of the residual obtained when plugging $u_{\mathrm{uv}}^{2, \delta}$ in place of the exact solution $u^{\delta}$ in problem (1.10).

3.2. Residual bounds. In this part, we establish a bound on the residual which can be characterized by the mapping $v \mapsto a^{\delta}\left(u_{\mathrm{uv}}^{2, \delta}-u^{\delta}, v\right)$. It clearly defines a continuous linear form on $H^{1}\left(\Omega^{\delta}\right)$ since $u_{\mathrm{uv}}^{2, \delta}-\exp \left(i \kappa_{\mathrm{S}} X\right)$ exponentially decays to zero as $X \rightarrow-\infty$ within the cavity.

3.2.1. Decomposition of the residual.

Lemma 3.2. The residual can be written in the following form:

$$
\begin{aligned}
& a^{\delta}\left(u_{\mathrm{uv}}^{2, \delta}-u^{\delta}, v\right)=a^{\delta}\left(u_{\text {out }}^{2, \delta},\left(1-\Phi_{\delta}\right) v\right) \\
& \quad-\ell_{\delta}\left(1-\Phi_{\delta}\right) v+a^{\delta}\left(u_{\mathrm{inn}}^{2, \delta}, \Phi_{\delta} v\right)+m^{\delta}\left(u_{\mathrm{out}}^{2, \delta}-u_{\mathrm{inn}}^{2, \delta}, v\right)
\end{aligned}
$$

with

$$
m^{\delta}(u, v)=\int_{\Omega^{\delta}}\left(u \nabla \Phi_{\delta} \cdot \nabla v-\nabla u \cdot v \nabla \Phi_{\delta}\right) d x d y .
$$

Proof. Using (1.11), we readily get the decomposition by an elementary argument. 
The residual is thus decomposed into three terms according to the various kinds of errors:

- an outer error: $a^{\delta}\left(u_{\text {out }}^{2, \delta},\left(1-\Phi_{\delta}\right) v\right)-\ell_{\delta}\left(1-\Phi_{\delta}\right) v$,

- an inner error: $a^{\delta}\left(u_{\mathrm{inn}}^{2, \delta}, \Phi_{\delta} v\right)$,

- an error due to the matching: $m^{\delta}\left(u_{\text {out }}^{2, \delta}-u_{\text {inn }}^{2, \delta}, v\right)$.

To bound the residual, we are thus led to get an estimate on each of these terms. In all of the sequel, $C$ indicates various constants, not the same in all instances, always independent of $\delta$.

3.2.2. The outer error. To bound the part of the residual related to the outer error, we prove that the outer expansion $u_{\text {out }}^{2, \delta}$ approximately solves the same equations as $u^{\delta}$ in the zone $|x|,|y|>\sqrt{\delta}$.

Lemma 3.3. The following estimates hold true:

$$
\begin{gathered}
\left\|\left(\partial_{x}^{2}+\kappa^{2}\right) u^{1,0}\right\|_{L^{\infty}(] \sqrt{\delta},+\infty[\times\{0\})} \leq \frac{C}{\delta^{3 / 4}}, \\
\left\|\left(\partial_{x}^{2}+\kappa^{2}\right) u^{2,0}\right\|_{L^{2}(] \sqrt{\delta},+\infty[\times\{0\})} \leq \frac{C|\ln \delta|}{\delta^{5 / 4}}, \\
\left\|\left(\partial_{x}^{2}+\kappa^{2}\right) u^{2,1}\right\|_{L^{2}\left(\left[\delta^{\frac{1}{2}},+\infty[\times\{0\})\right.\right.} \leq \frac{C|\ln \delta|}{\delta^{5 / 4}} .
\end{gathered}
$$

Proof. Using the second-order Bessel differential equations satisfied respectively by $H_{0}^{(1)}$ and $H_{1}^{(1)}$, as well as the expressions of their derivatives, and the asymptotic behaviors of the Hankel functions for small values of the argument (cf., e.g., 35]), we readily get

$$
\begin{aligned}
& \left(\partial_{x}^{2}+\kappa^{2}\right) H_{0}^{(1)}(\kappa x)=\underset{x \rightarrow 0}{O}\left(\frac{1}{x^{2}}\right), \\
& \left(\partial_{x}^{2}+\kappa^{2}\right) H_{1}^{(1)}(\kappa x)=\underset{x \rightarrow 0}{O}\left(\frac{1}{x^{3}}\right),
\end{aligned}
$$

which directly give

$$
\begin{aligned}
& \left\|\left(\partial_{x}^{2}+\kappa^{2}\right) H_{0}^{(1)}(\kappa x)\right\|_{L^{2}(] \sqrt{\delta},+\infty[\times\{0\})} \leq \frac{C}{\delta^{3 / 4}}, \\
& \left\|\left(\partial_{x}^{2}+\kappa^{2}\right) H_{1}^{(1)}(\kappa x)\right\|_{L^{2}(] \sqrt{\delta},+\infty[\times\{0\})} \leq \frac{C}{\delta^{5 / 4}} .
\end{aligned}
$$

In the same way, we also obtain from the very definition of $\mathbf{s}$ which contains the singularity of $w^{2,0}$ (cf. (2.94) and (2.95) )

$$
\left.\|\left(\partial_{x}^{2}+\kappa^{2}\right)(\mathrm{s})\right) \|_{\mathrm{L}^{2}(] \sqrt{\delta},+\infty[\times\{0\})} \leq \frac{C|\ln \delta|}{\delta^{5 / 4}} .
$$

Finally, since $w_{R} \in \mathrm{H}^{3-s}(\Omega)$ for all $s>0$, we have

$$
\left\|\left(\partial_{x}^{2}+\kappa^{2}\right) w_{R}\right\|_{L^{2}(] \sqrt{\delta},+\infty[\times\{0\})} \leq C .
$$

These bounds clearly yield the estimates stated in the lemma.

We can then get an estimate of the part of the residual relative to the outer error. 
Lemma 3.4. The following estimate holds true:

$$
\left|a^{\delta}\left(u_{\text {out }}^{2, \delta},\left(1-\Phi_{\delta}\right) v\right)-\ell_{\delta}\left(1-\Phi_{\delta}\right) v\right| \leq C \delta^{\frac{7}{4}}|\ln \delta|\|v\|_{\mathrm{H}^{1}\left(\Omega^{\delta}\right)} .
$$

Proof. In view of Lemma 2.1, the outer expansion $u_{\text {out }}^{2, \delta}$ satisfies

$$
\left\{\begin{array}{l}
\left(\Delta+\kappa^{2}\right) u_{\text {out }}^{2, \delta}=0 \text { in } \Omega, \\
\partial_{y} u_{\text {out }}^{2, \delta}\left(x, 0^{+}\right)=-\delta^{2} 1_{x>0} \pi\left(\partial_{x}^{2}+\kappa^{2}\right) u^{1,0}(x, 0) \text { for } x \neq 0, \\
u_{\text {out }}^{2, \delta}(x, y)=u_{\text {out }}^{2, \delta}\left(x, 0^{+}\right) \text {for } x>0 \text { and }-\pi \delta<y<0, \\
\left(\Delta+\kappa^{2}\right) u_{\text {out }}^{2, \delta}(x, y)=0 \text { for } x<0 \text { and }-\pi \delta<y<0, \\
\partial_{y} u_{\text {out }}^{2, \delta}\left(x, 0^{-}\right)=0 \text { for } x<0 \\
\partial_{y} u_{\text {out }}^{2, \delta}(x,-\pi \delta)=0 \text { for } x<0
\end{array}\right.
$$

Making use of Green's formula and taking into account that $u_{\text {out }}^{2, \delta}(x, y)=u_{\text {out }}^{2, \delta}(x, 0)$ and $\Phi_{\delta}(x, y)=\Phi_{\delta}(x, 0)$ for $x>0$ and $-\pi \delta<y<0$, we get

$$
\begin{aligned}
& a^{\delta}\left(u_{\mathrm{out}}^{2, \delta},\left(1-\Phi_{\delta}\right) v\right)-\ell_{\delta}\left(1-\Phi_{\delta}\right) v \\
& \quad=\delta^{2} \int_{0}^{+\infty}\left(\partial_{x}^{2}+\kappa^{2}\right) u^{1,0}(x, 0)\left(1-\Phi_{\delta}(x, 0)\right) \int_{-\pi}^{0}(v(x, 0)-v(x, \delta Y)) d Y d x \\
& \quad-\int_{0}^{+\infty}\left(\int_{-\pi \delta}^{0}\left(\partial_{x}^{2}+\kappa^{2}\right)\left(\delta^{2} u^{2,0}+\delta^{2} \ln \delta u^{2,1}\right)(x, 0)\left(1-\Phi_{\delta}(x, 0)\right) v(x, y) d y\right) d x .
\end{aligned}
$$

Noting that $\left|1-\Phi_{\delta}(x, y)\right| \leq 1$ and using the Cauchy-Schwarz inequality, we come to

$$
\begin{aligned}
& \mid a^{\delta}\left(u_{\text {out }}^{2, \delta},\left(1-\Phi_{\delta}\right) v\right)-\ell_{\delta}\left(1-\Phi_{\delta}\right) v \mid \\
& \leq \delta^{2}\left\|\left(\partial_{x}^{2}+\kappa^{2}\right) u^{1,0}\right\|_{\mathrm{L}^{2}(] \sqrt{\delta},+\infty[\times\{0\})}\left(\int_{0}^{+\infty}\left(\int_{-\pi}^{0}(v(x, 0)-v(x, \delta Y)) d Y\right)^{2} d x\right)^{\frac{1}{2}} \\
&+\delta^{\frac{1}{2}}\left\|\left(\partial_{x}^{2}+\kappa^{2}\right)\left(\delta^{2} u^{2,0}+\delta^{2} \ln \delta u^{2,1}\right)\right\|_{\mathrm{L}^{2}(] \sqrt{\delta},+\infty[\times\{0\})}\|v\|_{\mathrm{L}^{2}\left(\Omega_{\mathrm{L}}^{\delta}\right)} .
\end{aligned}
$$

Lemma 3.3 and the following estimates (cf. Lemma 6.2.1 in [22]) complete the proof:

$$
\begin{gathered}
\left(\int_{0}^{+\infty}\left(\int_{-\pi \delta}^{0}(v(x, 0)-v(x, y)) d y\right)^{2} d x\right)^{\frac{1}{2}} \leq C \delta^{\frac{1}{2}}\|v\|_{\mathrm{H}^{1}\left(\Omega^{\delta}\right)}, \\
\|v\|_{\mathrm{L}^{2}\left(\Omega_{\mathrm{L}}^{\delta}\right)} \leq C \delta^{\frac{1}{2}}\|v\|_{\mathrm{H}^{1}\left(\Omega^{\delta}\right)} .
\end{gathered}
$$

3.2.3. The inner error. To state the bounds on the residual related to the error in the inner zone, we need the following notation:

$$
B_{\eta}^{\infty}=\left\{(x, y) \in \mathbb{R}^{2}:|x|,|y|<\eta\right\} .
$$


Lemma 3.5. The following estimates hold true:

$$
\begin{array}{ll}
\left\|\widetilde{\Pi}^{n, m}\right\|_{L^{\infty}\left(\Omega \cap B_{2 \sqrt{\delta}}^{\infty}\right\}} \leq C|\ln \delta|, & \text { for } 0<m<n \leq 2, \\
\left\|\widetilde{\Pi}^{n, m}\right\|_{L^{\infty}\left(\Omega \cap B_{2 \sqrt{\delta}}^{\infty}\right)} \leq C, & \text { for } 0<n \leq 2, \\
\left\|\widetilde{\Pi}^{n, m}\right\|_{L^{\infty}\left(\Omega_{\mathrm{L} \cap B_{2 \sqrt{\delta}}^{\delta}}^{\infty} \leq C|\ln \delta|,\right.} \leq C, & \text { for } 0<m<n \leq 2, \\
\left\|\widetilde{\Pi}^{n, n}\right\|_{L_{\left(\infty \Omega_{\mathrm{L}}^{\delta} \cap B_{2 \sqrt{\delta}}^{\infty}\right)} \leq C,} \leq \text { for } 0<n \leq 2, \\
\left\|\widetilde{\Pi}^{n, m}\right\|_{L^{\infty}\left(\Omega_{\mathrm{C}}^{\delta} \cap B_{2 \sqrt{\delta}}^{\infty}\right)} \leq C \delta^{-\frac{1}{2}}, & \text { for } 0<m<n \leq 2, \\
\left\|\widetilde{\Pi}^{n, m}\right\|_{L^{\infty}\left(\Omega_{\mathrm{C}}^{\delta} \cap B_{2 \sqrt{\delta}}^{\infty}\right)} \leq C, & \text { for } 0<n \leq 2 .
\end{array}
$$

Proof. Inequalities (3.24), (3.26) and (3.28) are a direct consequence of the fact that $\Pi^{n, n}$ is constant. For $m<n$, noting that for $(x, y) \in B_{2 \sqrt{\delta}}^{\infty},|x / \delta|,|y / \delta|<2 / \sqrt{\delta}$, we use the behavior at infinity of $\Re(\ln \omega(X, Y))$ given by (2.43) and (2.44) to write

$$
\begin{aligned}
& \|\Re \ln \omega(x / \delta, y / \delta)\|_{L^{\infty}\left(\Omega \cap B_{2 \sqrt{\delta}}^{\infty}\right)} \leq C|\ln \delta|, \\
& \|\Re \ln \omega(x / \delta, y / \delta)\|_{L^{\infty}\left(\Omega_{\mathrm{L}}^{\delta} \cap B_{2 \sqrt{\delta}}^{\infty}\right)} \leq C|\ln \delta|, \\
& \|\Re \ln \omega(x / \delta, y / \delta)\|_{L^{\infty}\left(\Omega_{\mathrm{C}}^{\delta} \cap B_{2 \sqrt{\delta}}^{\infty}\right)} \leq C \delta^{-1 / 2} .
\end{aligned}
$$

The end of the proof follows these estimates in a straightforward way.

This leads us to the bound embodied in the following lemma.

LEmma 3.6. The part of the residual related to the error in the inner zone is bounded by

$$
\left|a^{\delta}\left(u_{\mathrm{inn}}^{2, \delta}, \Phi_{\delta} v\right)\right| \leq C \delta^{\frac{3}{2}}\|v\|_{\mathrm{H}^{1}\left(\Omega_{\delta}\right)} .
$$

Proof. From $\Pi^{0,0}=0$ and (2.37), we obviously get that all the coefficients of the inner expansion satisfy $\Delta_{X, Y} \Pi^{n, m}=0$ in $\widehat{\Omega}$ and $\partial_{\mathbf{n}} \Pi^{n, m}=0$ on $\partial \widehat{\Omega}$. Since $\Phi_{\delta}$ is compactly supported in $\mathbb{R}^{2}$, we can then write

$$
\int_{\Omega^{\delta}} \nabla u_{\mathrm{inn}}^{2, \delta} \cdot \nabla\left(\Phi_{\delta} v\right) d x d y=0, \quad \forall v \in \mathrm{H}^{1}\left(\Omega^{\delta}\right)
$$

Consequently, one has

$$
a^{\delta}\left(u_{\mathrm{inn}}^{2, \delta}, \Phi_{\delta} v\right)=-\kappa^{2} \int_{\Omega^{\delta}} u_{\mathrm{inn}}^{2, \delta} \Phi_{\delta} v d x d y .
$$

Since $\Phi_{\delta}$ is supported in $B_{2 \sqrt{\delta}}^{\infty}$, Young's inequalities give

$$
\begin{aligned}
& \left|a^{\delta}\left(u_{\mathrm{inn}}^{2, \delta}, \Phi_{\delta} v\right)\right| \leq C\left\|u_{\mathrm{inn}}^{2, \delta}\right\|_{\mathrm{L}_{\left(\Omega\left(\Omega \cap B_{2 \sqrt{\delta}}^{\infty}\right\}\right)}}\|v\|_{\mathrm{L}^{1}\left(\Omega \cap \cap B_{2 \sqrt{\delta}}^{\infty}\right)} \\
& \quad+C\left\|u_{\mathrm{inn}}^{2, \delta}\right\|_{\mathrm{L}^{\infty}\left(\Omega_{\mathrm{L}}^{\delta} \cap B_{2 \sqrt{\delta}}^{\infty}\right)}\|v\|_{\mathrm{L}^{1}\left(\Omega_{\mathrm{L}}^{\delta} \cap B_{2 \sqrt{\delta}}^{\infty}\right)}+C\left\|u_{\mathrm{inn}}^{2, \delta}\right\|_{L_{\left(\infty\left(\Omega_{\mathrm{C}}^{\delta} \cap B_{2 \sqrt{\delta}}^{\infty}\right)\right.}\|v\|_{\mathrm{L}^{1}\left(\Omega_{\mathrm{C}}^{\delta} \cap B_{2 \sqrt{\delta}}^{\infty}\right)} .} .
\end{aligned}
$$


Taking into account that $\Pi_{\delta}^{0,0} \equiv 0$, and using Lemma 3.5. we readily get the following bounds:

$$
\begin{aligned}
& \left\|u_{\mathrm{inn}}^{2, \delta}\right\|_{\mathrm{L}_{\left(\Omega\left(\Omega \cap B_{2 \sqrt{\delta}}^{\infty}\right\}\right)}} \leq C \delta|\ln \delta| \\
& \left\|u_{\mathrm{inn}}^{2, \delta}\right\|_{\mathrm{L}_{\left(\Omega_{\mathrm{L}}^{\infty} \cap B_{2 \sqrt{\delta}}^{\infty}\right)}} \leq C \delta^{\frac{1}{2}} \\
& \left\|u_{\mathrm{inn}}^{2, \delta}\right\|_{\mathrm{L}_{\left(\Omega_{\mathrm{C}}^{\infty} \cap B_{2 \sqrt{\delta}}^{\infty}\right)}} \leq C \delta^{\frac{1}{2}}
\end{aligned}
$$

The end of the proof can then be directly obtained from the following estimates established in Lemma 3.10 of [17]:

$$
\begin{aligned}
& \|v\|_{\mathrm{L}^{1}\left(\Omega \cap B_{2 \sqrt{\delta}}^{\infty}\right)} \leq C \delta|\ln \delta|^{\frac{1}{2}}\|v\|_{\mathrm{H}^{1}\left(\Omega^{\delta}\right)} \\
& \|v\|_{\mathrm{L}^{1}\left(\Omega_{\mathrm{L}}^{\delta} \cap B_{2 \sqrt{\delta}}^{\infty}\right)} \leq C \delta\|v\|_{\mathrm{H}^{1}\left(\Omega^{\delta}\right)} \\
& \|v\|_{\mathrm{L}^{1}\left(\Omega_{\mathrm{C}}^{\delta} \cap B_{2 \sqrt{\delta}}^{\infty}\right)} \leq C \delta\|v\|_{\mathrm{H}^{1}\left(\Omega^{\delta}\right)}
\end{aligned}
$$

3.2.4. The matching error. The part of the residual relative to the matching zone is written in terms of the difference $u_{\text {out }}^{2, \delta}-u_{\text {inn }}^{2, \delta}$ of the outer and the inner expansions of $u^{\delta}$ in the matching region $C_{\delta}^{\infty}$. Bounding this part of the residual is the less standard step in obtaining sharp estimates of the error. We now give some details on the way to obtain these bounds.

LEMma 3.7. The matching bilinear form can be bounded as follows:

$$
\left|m^{\delta}(u, v)\right| \leq C\left(\begin{array}{c}
\|u\|_{\mathrm{L}^{\infty}\left(\Omega \cap C_{\delta}^{\infty}\right)}+\delta^{\frac{1}{2}} \sqrt{|\ln (\delta)|}\|\nabla u\|_{\mathrm{L}^{\infty}\left(\Omega \cap C_{\delta}^{\infty}\right)}+ \\
\delta^{\frac{1}{4}}\|u\|_{\mathrm{L}^{\infty}\left(\Omega_{\mathrm{L}}^{\delta} \cap C_{\delta}^{\infty}\right)}+\delta^{\frac{1}{2}}\|\nabla u\|_{\mathrm{L}^{\infty}\left(\Omega_{\mathrm{L}}^{\delta} \cap C_{\delta}^{\infty}\right)}+ \\
\delta^{\frac{1}{4}}\|u\|_{\mathrm{L}^{\infty}\left(\Omega_{\mathrm{C}}^{\delta} \cap C_{\delta}^{\infty}\right)}+\delta^{\frac{1}{2}\|\nabla u\|_{\mathrm{L}^{\infty}\left(\Omega_{\mathrm{C}}^{\delta} \cap C_{\delta}^{\infty}\right)}}
\end{array}\right)\|v\|_{\mathrm{H}^{1}\left(\Omega^{\delta}\right)}
$$

Proof. We first decompose the bilinear form $m^{\delta}$ into three terms, each of them corresponding to one of the above zones:

$$
\begin{aligned}
m^{\delta}(u, v) & =\int_{\Omega}\left(u \nabla \Phi_{\delta} \cdot \nabla v-\nabla u \cdot \nabla \Phi_{\delta} v\right) d x d y+\int_{\Omega_{\mathrm{L}}^{\delta}}\left(u \nabla \Phi_{\delta} \cdot \nabla v-\nabla u \cdot \nabla \Phi_{\delta} v\right) d x d y \\
& +\int_{\Omega_{\mathrm{C}}^{\delta}}\left(u \nabla \Phi_{\delta} \cdot \nabla v-\nabla u \cdot \nabla \Phi_{\delta} v\right) d x d y
\end{aligned}
$$

The support of $\nabla \Phi_{\delta}$ is included in $C_{\delta}^{\infty}$. Therefore, due to the Young inequality, one gets

$$
\begin{aligned}
\left|m^{\delta}(u, v)\right| \leq \frac{C}{\delta}\left(\|u\|_{\mathrm{L}^{\infty}\left(\Omega \cap C_{\delta}^{\infty}\right)}\|\nabla v\|_{\mathrm{L}^{1}\left(\Omega \cap C_{\delta}^{\infty}\right)}+\|\nabla u\|_{\mathrm{L}^{\infty}\left(\Omega \cap C_{\delta}^{\infty}\right)}\|v\|_{\mathrm{L}^{1}\left(\Omega \cap C_{\delta}^{\infty}\right)}\right. \\
+\|u\|_{\mathrm{L}^{\infty}\left(\Omega_{\mathrm{L}}^{\delta} \cap C_{\delta}^{\infty}\right)}\|\nabla v\|_{\mathrm{L}^{1}\left(\Omega_{\mathrm{L}}^{\delta} \cap C_{\delta}^{\infty}\right)}+\|\nabla u\|_{\mathrm{L}^{\infty}\left(\Omega_{\mathrm{L}}^{\delta} \cap C_{\delta}^{\infty}\right)}\|v\|_{\mathrm{L}^{1}\left(\Omega_{\mathrm{L}}^{\delta} \cap C_{\delta}^{\infty}\right)} \\
\left.+\|u\|_{\mathrm{L}^{\infty}\left(\Omega_{\mathrm{C}}^{\delta} \cap C_{\delta}^{\infty}\right)}\|\nabla v\|_{\mathrm{L}^{1}\left(\Omega_{\mathrm{C}}^{\delta} \cap C_{\delta}^{\infty}\right)}+\|\nabla u\|_{\mathrm{L}^{\infty}\left(\Omega_{\mathrm{C}}^{\delta} \cap C_{\delta}^{\infty}\right)}\|v\|_{\mathrm{L}^{1}\left(\Omega_{\mathrm{C}}^{\delta} \cap C_{\delta}^{\infty}\right)}\right) .
\end{aligned}
$$


The proof can be completed using the following estimates, which can be found in [22]:

$$
\begin{cases}\|\nabla v\|_{\mathrm{L}^{1}\left(\Omega \cap C_{\delta}^{\infty}\right)} \leq C \delta^{\frac{1}{2}}\|v\|_{\mathrm{H}^{1}\left(\Omega^{\delta}\right)}, & \|v\|_{\mathrm{L}^{1}\left(\Omega \cap C_{\delta}^{\infty}\right)} \leq C \delta|\ln \delta|^{\frac{1}{2}}\|v\|_{\mathrm{H}^{1}\left(\Omega^{\delta}\right)}, \\ \|\nabla v\|_{\mathrm{L}^{1}\left(\Omega_{\mathrm{L}}^{\delta} \cap C_{\delta}^{\infty}\right)} \leq C \delta^{\frac{3}{4}}\|v\|_{\mathrm{H}^{1}\left(\Omega^{\delta}\right)}, & \|v\|_{\mathrm{L}^{1}\left(\Omega_{\mathrm{L}}^{\delta} \cap C_{\delta}^{\infty}\right)} \leq C \delta\|v\|_{\mathrm{H}^{1}\left(\Omega^{\delta}\right)}, \\ \|\nabla v\|_{\mathrm{L}^{1}\left(\Omega_{\mathrm{C}}^{\delta} \cap C_{\delta}^{\infty}\right)} \leq C \delta^{\frac{3}{4}}\|v\|_{\mathrm{H}^{1}\left(\Omega^{\delta}\right)}, & \|v\|_{\mathrm{L}^{1}\left(\Omega_{\mathrm{C}}^{\delta} \cap C_{\delta}^{\infty}\right)} \leq C \delta\|v\|_{\mathrm{H}^{1}\left(\Omega^{\delta}\right)} .\end{cases}
$$

In the construction of the asymptotic expansion, we have expressed the outer expansion in terms of the fast variables and expand it to match it to the inner one. The following lemma now shows how the expressions of the asymptotic behavior of the inner expansion in terms of the slow variables can be connected to the outer one. It constitutes an important step in obtaining a bound on the error due to the matching procedure.

Lemma 3.8. Let $U_{2}^{n, m}(0 \leq m \leq n \leq 2)$ be the functions given in (2.96) describing the behavior at infinity of the coefficients of the second-order inner asymptotic expansion. Then, we have

$$
\sum_{0 \leq m \leq n \leq 2} \delta^{n} \ln ^{m} \delta U_{2}^{n, m}(x / \delta, y / \delta)=\sum_{0 \leq m \leq n \leq 2} \delta^{n} \ln ^{m} \delta u_{2}^{n, m}(x, y)
$$

with $u_{2}^{n, m}=C_{0}^{1,0} \widetilde{u}_{2}^{n, m}$ given by

$$
\left\{\begin{array}{l}
\widetilde{u}_{2}^{0,0}=\widetilde{u}_{2}^{1,1}=\widetilde{u}_{2}^{2,2}=0, \\
\widetilde{u}_{2}^{1,0}(x, y)=\frac{2 i}{\pi} \ln r+\gamma_{\kappa}, \\
\widetilde{u}_{2}^{2,0}(x, y)=\frac{2 i}{\pi}\left(\mathrm{s}_{-1}(r, \theta)+\mathrm{s}_{1}(r, \theta)+r^{2,0}(0,0)\right) \\
\quad \quad-i \kappa\left(\gamma_{\kappa}-\frac{2 i}{\pi}\right) \ln r-\frac{\pi \kappa}{2}\left(\gamma_{\kappa}-\frac{2 i}{\pi}\right)-\frac{2 i}{\pi} \frac{\cos \theta}{r}, \\
\widetilde{u}_{2}^{2,1}(x, y)=-i \kappa \gamma_{\kappa}+\frac{2 i}{\pi} \frac{\cos \theta}{r}+\frac{2 \kappa}{\pi} \ln r .
\end{array}\right.
$$

Moreover, the nonzero coefficients are linked to those of the outer expansion as follows:

$$
\left\{\begin{array}{lll}
\left(u^{1,0}-u_{2}^{1,0}\right)(x, y)=O_{r \rightarrow 0}\left(r^{2} \ln r\right), & \nabla\left(u^{1,0}-u_{2}^{1,0}\right)(x, y)=O_{r \rightarrow 0}(r \ln r), \\
\left(u^{2,0}-u_{2}^{2,0}\right)(x, y)=O_{r \rightarrow 0}(r \ln r), & \nabla\left(u^{2,0}-u_{2}^{2,0}\right)(x, y)=O_{r \rightarrow 0}(\ln r), \\
\left(u^{2,1}-u_{2}^{2,1}\right)(x, y)=O_{r \rightarrow 0}(r \ln r), & \nabla\left(u^{2,1}-u_{2}^{2,1}\right)(x, y)=O_{r \rightarrow 0}(\ln r) .
\end{array}\right.
$$

Proof. The proof can be obtained by a simple check from the formulas defining the inner and the outer asymptotic expansions and the asymptotic behavior of the Hankel functions recalled in (2.33), (2.34) and (2.35).

Lemma 3.9. Defining

$$
u_{2, \text { out }}^{2, \delta}=\sum_{0 \leq m \leq n \leq 2} \delta^{n} \ln ^{m} \delta u_{2}^{n, m},
$$

the above lemma immediately yields

$$
\left\|u_{\text {out }}^{2, \delta}-u_{2, \text { out }}^{2, \delta}\right\|_{L^{\infty}\left(\Omega \cap C_{\delta}^{\infty}\right)}+\delta^{1 / 2}\left\|\nabla\left(u_{\text {out }}^{2, \delta}-u_{2, \text { out }}^{2, \delta}\right)\right\|_{L^{\infty}\left(\Omega \cap C_{\delta}^{\infty}\right)} \leq C \delta^{2}|\ln \delta| .
$$

We proceed similarly for the inner error. 
LEmma 3.10. The coefficients $\Pi^{n, m}$ are either equal to the corresponding intermediate functions $U_{2}^{n, m}$ or satisfy

$$
\begin{gathered}
\left\{\begin{array}{l}
\left(\Pi^{1,0}-U_{2}^{1,0}\right)(X, Y)=O_{R \rightarrow \infty}\left(\frac{\ln ^{2} R}{R^{2}}\right), \\
\nabla_{X, Y}\left(\Pi^{1,0}-U_{2}^{1,0}\right)(X, Y)=O_{R \rightarrow \infty}\left(\frac{\ln ^{2} R}{R^{3}}\right),
\end{array}\right. \\
\left\{\begin{array}{l}
\left(\Pi^{2, m}-U_{2}^{2, m}\right)(X, Y)=O_{R \rightarrow \infty}\left(\frac{\ln R}{R}\right), \\
\nabla_{X, Y}\left(\Pi^{2, m}-U_{2}^{2, m}\right)(X, Y)=O_{R \rightarrow \infty}\left(\frac{\ln R}{R^{2}}\right)
\end{array} \quad(m=0,1) .\right.
\end{gathered}
$$

Proof. From (2.83) and (2.96), we can express $\Pi^{1,0}-U_{2}^{1,0}$ as follows:

$$
\Pi^{1,0}-U_{2}^{1,0}=b^{1,2} \Re\left(\ln \omega-\left(\ln (-Z)-\frac{\ln (-Z)+1}{Z}\right)\right) .
$$

Similarly, we can write

$$
\Pi^{2, m}-U_{2}^{2, m}=b^{2, m} \Re(\ln \omega-\ln (-Z)) .
$$

The first part of estimates (3.37) and (3.38) directly follows from (2.43), those on the gradients from the behavior of

$$
\partial_{Z}(\ln \omega-(\ln (-Z)-(\ln (-Z)+1) / Z))=\frac{\partial_{Z} \omega}{\omega}-\frac{1}{Z}\left(1+\frac{\ln (-Z)}{Z}\right)
$$

and

$$
\partial_{Z}(\ln \omega-\ln (-Z))=\frac{\partial_{Z} \omega}{\omega}-\frac{1}{Z} .
$$

From (2.42), we get $\partial_{Z} \omega / \omega=1 /(1-\omega)=(1 / Z)(1 /(1-(\ln \omega) / Z)$, which directly completes the estimates.

Lemma 3.11. Defining

$$
u_{2, \text { inn }}^{2, \delta}(x, y)=\sum_{0 \leq m \leq n \leq 2} \delta^{n} \ln ^{m} \delta U_{2}^{n, m}(x / \delta, y / \delta),
$$

we have

$$
\left\|u_{\mathrm{inn}}^{2, \delta}-u_{2, \mathrm{inn}}^{2, \delta}\right\|_{L^{\infty}\left(\Omega \cap C_{\delta}^{\infty}\right)}+\delta^{1 / 2}\left\|\nabla\left(u_{\mathrm{inn}}^{2, \delta}-u_{2, \mathrm{inn}}^{2, \delta}\right)\right\|_{L^{\infty}\left(\Omega \cap C_{\delta}^{\infty}\right)} \leq C \delta^{2} \ln ^{2} \delta .
$$

Since the only nonzero coefficients $u_{\mathrm{L}}^{n, m}$ corresponding to the outer expansion in the thin layer are given by (2.91) and (2.98), the bounds corresponding to the thin layer are a direct consequence of those of the half-plane.

Lemma 3.12. Extending $u_{2, \text { out }}^{2, \delta}$ and $u_{2, \text { inn }}^{2, \delta}$ in $\Omega_{\mathrm{L}}^{\delta}$ by $u_{2, \text { out }}^{2, \delta}(x, y)=u_{2, \text { out }}^{2, \delta}(x, 0)$ and $u_{2, \text { inn }}^{2, \delta}(x, y)=u_{2, \text { inn }}^{2, \delta}(x, 0)$ for $-\pi \delta \leq y \leq 0$, we get the same bounds as (3.36) and (3.42) in which $\Omega$ is replaced by $\Omega_{\mathrm{L}}^{\delta}$.

We now repeat the above procedure for the cavity.

Lemma 3.13. Similarly to (3.33), we define $u_{2, \mathrm{C}}^{n, m}(x, y)$ through the following identification:

$$
\sum_{0 \leq m \leq n \leq 2} \delta^{n} \ln ^{m} \delta U_{\mathrm{C}}^{n, m}(x / \delta, y / \delta)=\sum_{0 \leq m \leq n \leq 2} \delta^{n} \ln ^{m} \delta u_{2, \mathrm{C}}^{n, m}(x, y) .
$$


These functions are given by

$$
u_{2, \mathrm{C}}^{n, m}(x, y)=a^{n, m}-b^{n, m}+b^{n+1, m} x
$$

with the convention that any coefficient $a^{n, m}$ or $b^{n, m}$ corresponding to an index $(n, m)$ outside the range $0 \leq m \leq n \leq 2$ is zero. Note that within this range, those of these coefficients which are not explicitly given above are zero. Moreover, the functions of the slow variable $u_{2, \mathrm{C}}^{n, m}(x, y)$ are linked to those of the outer expansion in the cavity as follows:

$$
\left\{\begin{array}{l}
u_{\mathrm{C}}^{n, m}(x, y / \delta)-u_{2, \mathrm{C}}^{n, m}(x, y)=\underset{x \rightarrow 0}{O}\left(x^{3-n}\right), \\
\nabla\left(u_{\mathrm{C}}^{n, m}(x, y / \delta)-u_{2, \mathrm{C}}^{n, m}(x, y)\right)=\underset{x \rightarrow 0}{O}\left(x^{2-n}\right)
\end{array}(0 \leq n \leq 2) .\right.
$$

Proof. The first part only repeats the matching rules within the cavity. To prove (3.45), we first consider the case corresponding to $n=0$ and $m=0$. Using (2.79) and (3.44), we can write

$$
u_{\mathrm{C}}^{0,0}(x, y / \delta)-u_{2, \mathrm{C}}^{0,0}(x, y)=2 i \sin \left(\kappa_{\mathrm{s}} x\right)-b^{1,0} x .
$$

From (2.83), we have $b^{1,0}=2 i \kappa$ and easily get (3.45) for $n=0$. The rest of the proof is a direct consequence of the connection between the coefficients $R^{n, m}$ of the asymptotic expansion of the reflection coefficient and the coefficients $a^{n, m}$ and $b^{n, m}$.

The inner error inside the cavity is exponentially decreasing as $X \rightarrow-\infty$.

LEMmA 3.14. The following estimates hold true:

$$
\begin{aligned}
\left(U_{\mathrm{C}}^{n, m}-\Pi^{n, m}\right)(X, Y) & =\underset{X \rightarrow-\infty}{O}\left(|X|^{-p}\right), \\
\nabla\left(U_{\mathrm{C}}^{n, m}(X, Y)-\Pi^{n, m}\right)(X, Y) & =\underset{X \rightarrow-\infty}{O}\left(|X|^{-p}\right),
\end{aligned}
$$

for $-\pi<Y<0,0 \leq m \leq n \leq 2$ and any positive integer $p$.

Proof. Making use of (2.97), we reduce the asymptotic behavior to the following:

$$
\Re \ln \omega-(X-1)=\underset{X \rightarrow-\infty}{O}\left(|X|^{-p}\right) \text { and } \nabla(\Re \ln \omega-(X-1))=\underset{X \rightarrow-\infty}{O}\left(|X|^{-p}\right) .
$$

The first of these is given in (2.44). For the derivatives, it is sufficient to observe that $\partial_{X}=\partial_{Z}$ and $\partial_{Y}=i \partial_{Z}$ to reduce the statement to

$$
\frac{\partial_{Z} \omega}{\omega}-1=\underset{X \rightarrow-\infty}{O}\left(|X|^{-p}\right) .
$$

To complete the proof, just note that we have seen above that $\partial_{Z} \omega / \omega=1 /(1-\omega)$.

In the same way as for the half-space and the substrate zone, we directly deduce from the above asymptotic behaviors a bound on the matching error.

Lemma 3.15. Similarly to the case of the half-plane, we define

$$
u_{\mathrm{C}, \text { out }}^{2, \delta}=\sum_{0 \leq m \leq n \leq 2} \delta^{n} \ln ^{m} \delta u_{2, \mathrm{C}}^{n, m}
$$

and

$$
u_{\mathrm{C}, \text { inn }}^{2, \delta}(x, y)=\sum_{0 \leq m \leq n \leq 2} \delta^{n} \ln ^{m} \delta U_{\mathrm{C}}^{n, m}(x / \delta, y / \delta) .
$$


The following bound holds true:

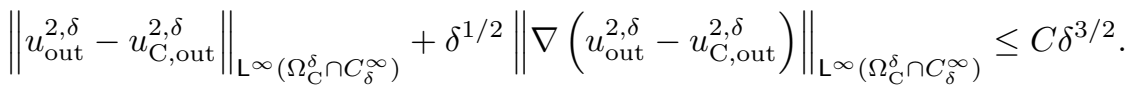

The part of the error related to the inner matching decreases faster than any power $\ell$ of $\delta$ :

$$
\left\|u_{\text {inn }}^{2, \delta}-u_{\mathrm{C}, \text { inn }}^{2, \delta}\right\|_{L^{\infty}\left(\Omega_{\mathrm{C}}^{\delta} \cap C_{\delta}^{\infty}\right)}+\delta^{1 / 2}\left\|\nabla\left(u_{\text {inn }}^{2, \delta}-u_{\mathrm{C}, \text { inn }}^{2, \delta}\right)\right\|_{L^{\infty}\left(\Omega_{\mathrm{C}}^{\delta} \cap C_{\delta}^{\infty}\right)} \leq C \delta^{\ell} .
$$

We then come to the main lemma of this part giving an estimate of the part of the residual due to the matching error whose proof directly follows from (3.32) and the bounds given in the above lemmas.

Lemma 3.16. The part of the residual due to the matching error can be bounded as follows:

$$
\left|m^{\delta}\left(u_{\mathrm{out}}^{2, \delta}-u_{\mathrm{inn}}^{2, \delta}, v\right)\right| \leq C \delta^{3 / 2}\|v\|_{\mathrm{H}^{1}\left(\Omega^{\delta}\right)}, \forall v \in \mathrm{H}^{1}\left(\Omega^{\delta}\right)
$$

3.2.5. Justification of the cavity model. Collecting all the above bounds, we first get the following error estimate as a straightforward consequence of the stability property (1.14) and Lemmas 3.4, 3.6, and 3.16, and then we readily obtain the following bound for the uniformly valid approximation of the solution to problem (1.10).

Lemma 3.17. Function $u_{\mathrm{uv}}^{2, \delta}$ given in (3.2) is an approximation of $u^{\delta}$ to the solution to problem (1.10) satisfying

$$
\left\|u_{\mathrm{uv}}^{2, \delta}-u^{\delta}\right\|_{\mathrm{H}^{1}\left(\Omega^{\delta}\right)} \leq C \delta^{3 / 2} .
$$

This lemma permits us to prove the main result of this study, that is, the justification that the solution delivered by the cavity model yields an approximate value for the actual field radiated by a patch antenna.

Theorem 3.18. Let $\varrho$ be a fixed positive real. There exists a constant $C$ independent of $0<\delta \leq \varrho /\left(4 \pi^{2}\right)$ such that

$$
\left\|u^{\delta}-\delta C_{0}^{1,0} H_{0}\right\|_{\mathrm{H}^{1}(\Omega \cap\{r>\varrho\})} \leq C \delta^{3 / 2} .
$$

Proof. For such $\delta, u_{\text {uv }}^{2, \delta}=u_{\text {out }}^{2, \delta}$ in $\Omega \cap\{r>\varrho\}$. We thus have

$$
\begin{aligned}
\left\|u^{\delta}-\delta C_{0}^{1,0} H_{0}\right\|_{\mathrm{H}^{1}(\Omega \cap\{r>\varrho\})} \leq & \left\|u^{\delta}-u_{\text {out }}^{2, \delta}\right\|_{\mathrm{H}^{1}(\Omega \cap\{r>\varrho\})} \\
& +\delta^{2} \sum_{m=0}^{2}\left|\ln ^{m} \delta\right|\left\|u^{2, m}\right\|_{\mathrm{H}^{1}(\Omega \cap\{r>\varrho\})} .
\end{aligned}
$$

Since $u^{2, m} \in \mathrm{H}^{1}(\Omega \cap\{r>\varrho\})$, this establishes the theorem.

4. Concluding remarks. In a simplified version, but still incorporating the main features of the radiation properties of a microstrip antenna, we have obtained a rigorous justification of a key heuristic assumption at the basis of the cavity model for patch antennas. This justification is indeed supported by an error bound. This error bound is not optimal, but the authors are convinced that the optimal one is true. Actually, the optimal bound can be established simply by pushing the expansion up to order three. 
However, the calculations and the bounds to do this are very intricate without leading to a better understanding of this model.

Acknowledgement. The authors would like to aknowledge the comments of the anonymous referee which contributed to improving the final appearance of the paper.

\section{REFERENCES}

[1] C. A. Balanis, Antenna theory: Analysis and design, third edition, Wiley-Interscience, Hoboken, New Jersey, 2005.

[2] G. Caloz, M. Costabel, M. Dauge, and G. Vial, Asymptotic expansion of the solution of an interface problem in a polygonal domain with thin layer, Asymptotic Analysis 50 (2006), no. 1/2, 121-173. MR2286939 (2009d:35048)

[3] K. R. Carver and J. W. Mink, Microstrip Antenna Technology, IEEE Trans. Antennas Propagation 29 (1981), no. 1, 2-24.

[4] D. C. Chang and E. F. Kuester, Total and partial reflection from the end of a parallel-plate waveguide with an extended dielectric slab, Radio Science 16 (1981), no. 1, 1-13.

[5] J. Chazarain and A. Piriou, Introduction to the theory of partial differential equations, NorthHolland Publishing Co., Amsterdam and New York, 1982. MR678605 (83j:35001)

[6] W. C. Chew and J. A. Kong, Asymptotic formula for the capacitance of two oppositely charged discs, Math. Proc. Camb. Phil. Soc. 89 (1981), 373-384. MR600251 (82h:78008)

[7] _ Microstrip capacitance for a circular disk through matched asymptotic expansions, SIAM J. Appl. Math. 42 (1982), 302-317. MR650226 (83k:78011)

[8] C.-L. Chi and N. G. Alexopoulos, An efficient numerical approach for modeling microstrip-type antennas, IEEE Transactions on Antennas and Propagation 38 (1990), no. 9, 1399-1404.

[9] J. Cousteix and J. Mauss, Asymptotic analysis and Boundary Layers, Springer-Verlag, New York, 2007. MR2301806 (2008a:76125)

[10] M. Dauge, Elliptic boundary value problems on corner domains, Lecture Notes in Mathematics, vol. 1341, Springer-Verlag, Berlin, 1988. MR.0961439 (91a:35078)

[11] R. W. Dearnley and A. R. F. Barel, A comparison of models to determine the resonant frequencies of a rectangular microstrip antenna, IEEE Transactions on Antennas and Propagation 37 (1989), no. $1,114-118$.

[12] M. Van Dyke, Perturbation Methods in Fluid Mechanics, annoted edition, The Parabolic Press, Stanford, California, 1975. MR0416240(54:4315)

[13] W. Eckhaus, Matched Asymptotic Expansions and Singular Perturbations, North-Holland Mathematics Studies, vol. 6, North-Holland Publishing Company, Amsterdam and London, 1973. MR 0670800 (58:32369)

[14] A. M. Il'in, Matching of asymptotic expansions of solutions of Boundary Value Problems, American Mathematical Society, Providence, RI, 1992. MR.1182791 (93g:35016)

[15] J.-M. Jin, Z. Lou, Y.-J. Li, N. W. Riley, and D. J. Riley, Finite element analysis of complex antennas and arrays, IEEE Transactions on Antennas and Propagation 56 (2008), no. 8, 22222240. MR 2444885

[16] Jianming Jin, The finite element method in electromagnetics, second edition, John Wiley \& Sons, New York, 2002. MR1903357 (2004b:78019)

[17] P. Joly and S. Tordeux, Asymptotic analysis of an approximate model for time harmonic waves in media with thin slots, M2AN Math. Model. Numer. Anal. 40 (2006), no. 1, 63-97. MR2223505 (2007b:35067)

[18] Matching of Asymptotic Expansions for Wave Propagation in Media with Thin Slots I: The Asymptotic Expansion, Multiscale Modeling and Simulation: A SIAM Interdisciplinary Journal 5 (2006), no. 1, 304-336. MR2221320 (2007e:35041)

[19] E. F. Kuester, R. T. Johnk, and D. C. Chang, The thin-substrate approximation for reflection from the end of a slab-loaded parallel-plate waveguide with application to microstrip patch antennas., IEEE Transactions on Antennas and Propagation AP-30 (1982), no. 5, 910-917.

[20] N. Lebedev, Special functions and their applications, Prentice-Hall Inc., Englewood-Cliffs, New Jersey, 1965. MR0174795 (30:4988) 
[21] N. N. Lebedev, The electric field at the edge of a plane condenser containing a dielectric, Sov. Phys. Tech. Phys. 28 (1958), no. 3, 1234-1243. MR0103703 (21:2469)

[22] A. Makhlouf, Justification et Amélioration de Modèles d'Antennes Patch par la Méthode des Développements Asymptotiques Raccordés, Ph.D. thesis, INSA Toulouse, 2008.

[23] T. M. Martinson and E. F. Kuester, Accurate analysis of arbitrarily ShapedPatch resonators on thin substrates, IEEE Transactions on Microwave Theory and Technique 36 (1988), no. 2, 324-331.

[24] IEEE Transactions on Atennas and Propagation 37 (1989), no. 4, 413-417. MR991012 (90c:78022)

[25] __ A generalized edge boundary condition for open microstrip structures, Journal of Electromagnetic Waves and Applications 4 (1990), no. 4, 273-295.

[26] V. Maz'ya, S. Nazarov, and B. Plamenevskij, Asymptotic theory of elliptic boundary-value problems in singularly perturbed domains, Birkhäuser, Basel, 2000. MR.1779977(2001e:35044a) MR1779978 (2001e:35044b)

[27] P. McIver and A. D. Rawlins, Two-dimensional wave-scattering problems involving parallel-walled ducts, Quart. J. Mech. Appl. Math. 46 (1993), no. 1, 89-116. MR1222382 (93m:76009)

[28] J. R. Mosig and F. E. Gardiol, General Integral Equation Formulation for Microstrip Antennas and Scatterers, Proc. IEE 132 (1985), no. 7, 424-432.

[29] S. A. Nazarov and B. A. Plamenevsky, Elliptic problems in domains with piecewise smooth boundaries, De Gruyter Expositions in Mathematics, vol. 13, Walter de Gruyter \& Co., Berlin, 1994. MR 1283387 (95h:35001)

[30] J.-C. Nédélec, Acoustic and electromagnetic equations: Integral representations for harmonic problems, Springer, Berlin, 2001. MR1822275 (2002c:35003)

[31] E. Newman and P. Tulyathan, Analysis of microstrip antennas using moment methods, IEEE Transactions on Antennas and Propagation 29 (1981), no. 1, 47-53.

[32] B. Noble, Methods based on the Wiener-Hopf Technique for the solution of partial differential equations, Pergamon Press, London, New York, Paris, Los Angeles, 1958. MR0102719 (21:1505)

[33] M. Taylor, Partial differential equations I, basic theory, Springer-Verlag, New York, 1996. MR.1395148 (98b:35002b)

[34] S. Tordeux, Méthodes asymptotiques pour la propagation des ondes dans les milieux comportant des fentes, Ph.D. thesis, Université Versailles-Saint-Quentin en Yvelines, 2004.

[35] Z. X. Wang and D. R. Guo, Special Functions, World Scientific Publishing Co. Inc., Teaneck, New Jersey, 1989. MR1034956 (91a:33001)

[36] G. N. Watson, A treatise on the theory of Bessel functions. Reprint of the second (1944) edition. Cambridge Mathematical Library, Cambridge University Press, Cambridge, 1995. MR1349110 (96i:33010)

[37] C. H. Wilcox, Scattering theory for the d'Alembert equation in exterior domains, vol. 442, SpringerVerlag, Berlin, 1975. MR0460927 (57:918) 